\title{
Journal of Engineering Mathematics \\ Free vibration analysis of functionally graded beams with non-uniform cross-section using the differential transform method \\ --Manuscript Draft--
}

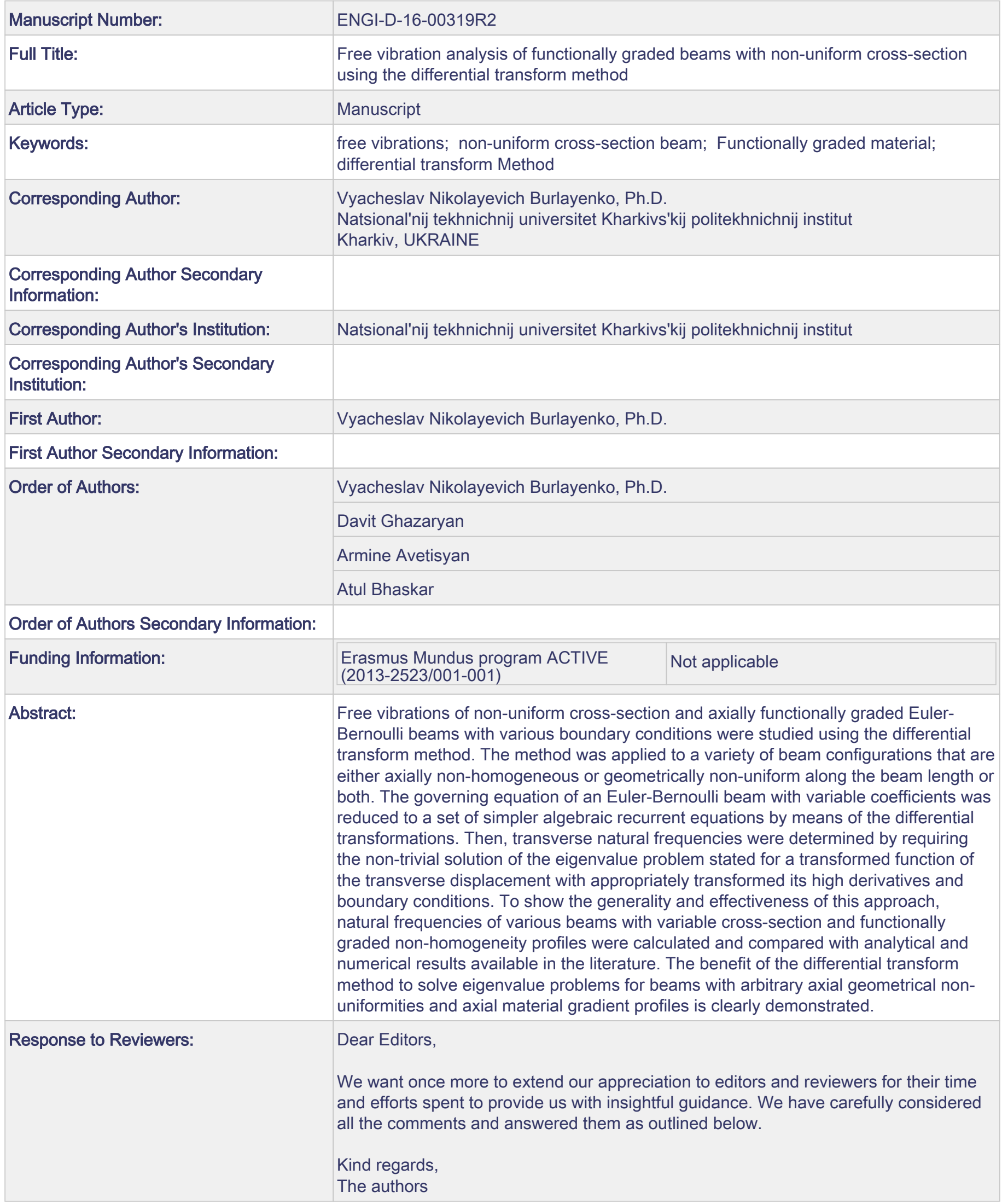




\title{
Free vibration analysis of functionally graded beams with non-uniform cross-section using the differential transform method
}

\author{
Davit Ghazaryan $^{\mathrm{a}, \mathrm{c}}$, Vyacheslav N. Burlayenko ${ }^{\mathrm{b}, \mathrm{c}, *}$, Armine Avetisyan $^{\mathrm{a}}$, Atul \\ Bhaskarc $^{c}$ \\ ${ }^{a}$ Department of Information Technology and Automation, National Polytechnic University \\ of Armenia, 105 Teryan Str., Yerevan 0009, Republic of Armenia \\ ${ }^{b}$ Department of Applied Mathematics, National Technical University 'KhPI', 2 Kyrpychova \\ Str., Kharkiv 61002, Ukraine \\ ${ }^{c}$ Faculty of Engineering and the Environment, University of Southampton, Southampton
} SO16 $7 Q F, U K$

\begin{abstract}
Free vibrations of non-uniform cross-section and axially functionally graded (AFG) Euler-Bernoulli beams with various boundary conditions were studied using the differential transform method. The method was applied to a variety of beam configurations that are either axially non-homogeneous or geometrically non-uniform along the beam length or both. The governing equation of an Euler-Bernoulli beam with variable coefficients was reduced to a set of simpler algebraic recurrent equations by means of the differential transformations. Then, transverse natural frequencies were determined by requiring the non-trivial solution of the eigenvalue problem stated for a transformed function of the transverse displacement with appropriately transformed its high derivatives and boundary conditions. To show the generality and effectiveness of this approach, natural frequencies of various beams with variable cross-section and functionally graded non-homogeneity profiles were calculated and compared with analytical and numerical results available in the literature. The benefit of the differential transform method to solve eigenvalue problems for beams with
\end{abstract}

\footnotetext{
* Corresponding author

Email addresses: D.Ghazaryan@pua.am, D.Ghazaryan@soton.ac.uk (Davit Ghazaryan), burlayenko@kpi.kharkov.ua, V.Burlayenko@soton.ac.uk (Vyacheslav N. Burlayenko), A.Avetisyan@pua.am (Armine Avetisyan), A.Bhaskar@soton.ac.uk (Atul Bhaskar)
} 
arbitrary axial geometrical non-uniformities and axial material gradient profiles is clearly demonstrated.

Keywords: free vibrations, non-uniform cross-section beam, functionally graded material, differential transform method

\section{Introduction}

Natural frequencies are fundamental to the understanding of the dynamics of mechanical systems. Their knowledge is required for analysing and designing a structure in dynamic environment. Contemporary in-service conditions impose additional requirements on the properties of materials used in structures. This is particularly true for structures that have to perform under extremely severe thermal loading and adverse environment. In this regard, functionally graded materials (FGM), a type of composite materials, are able to withstand high temperatures, thermal fracture and corrosion 1 14. Therefore, the vibration analysis of FGM structures is of critical importance from the standpoint of their safety and effective exploitation.

Because beams are the basic structural elements in engineering constructions on the one hand and the simplest models for theoretical research on the other hand, free vibration analysis of FGM beams has been extensively studied and continues to receive much attention in the literature. Many studies have been carried out on vibrations of beams containing a material gradient in the thickness direction. Various assumptions on deformation of beams and different approaches used for obtaining exact analytical and approximate numerical solutions have been reported. The comparison between different beam theories being exploited for natural frequency analysis of FGM beams is given in [5].

Analytical solutions for FGM beams with axially graded material properties are difficult to obtain. The complexity of the analysis lies in the presence of variable coefficients in the governing beam equation caused by the dependence

of material parameters on the axial coordinate. From the mathematical point of view this issue is similar to the problem, in which the terms of governing beam 
equation are functions of the axial coordinate due to variable cross-sectional area and flexural rigidity along the beam length. In the past this problem received a wide consideration among many researchers; see e.g. [6]. It is well-known that exact solutions of this problem can be obtained only for a few special cross-sectional profiles and boundary conditions. Free vibration analysis solved for beams with continuously and stepped varying cross-sectional parameters has been reviewed in recent publications, e.g. [7 9]. Both analytical solutions in terms of special functions including Bessel functions, hypergeometric series, power series, Bernstein polynomials and approximate solutions obtained by using Rayleigh-Ritz approach, finite element method, dynamic stiffness method, differential quadrature method, differential transform method have been reported there. A series of analytical solutions for prismatic beams with inhomogeneous material parameters prescribed in the form of certain polynomials has been obtained in [10, 11]. Li [12] was able to derive an analytical solution in terms of Bessel functions for the free vibration problem of beams containing determined both an axial geometrical non-uniformity and axial mass and shear stiffness distributions. Another analytical solution for natural frequencies of FGM beams is shown in [13], where the authors have considered exponential material gradient in the beam thickness direction and arbitrary variable crosssection along the beam length. Exact solutions of the free vibration problem for FGM beams with material profiles and cross-sectional parameters varying exponentially in the axial direction have been deduced in [14, 15], where assumptions of Euler-Bernoulli and Timoshenko beam theories have been applied respectively.

Apart from analytical solutions for analysing limited classes of beams satisfying defined assumptions of inhomogeneity and non-uniformity, many numerical approaches have been developed. Some of researchers have used the finite element method (FEM) for AFGM beams with arbitrary varying cross-section using appropriate homogeneous finite elements, e.g. in [16, 17] or developing special graded finite elements, e.g. in [18]. In [19] the authors studied the free vibration of beams with axial material gradation and non-uniform cross-section 
by transforming the governing differential equation with variable coefficients into Fredholm integral equations. The natural frequencies were determined by requiring that the resulting Fredholm equation has non-trivial solutions. The natural frequencies of non-uniform and AFGM beams with various boundary conditions and cross-sectional parameters have been calculated by using Haar wavelets in 20]. The differential quadrature method has been employed for studying free vibrations of tapered homogeneous and AFGM rotating cantilever beams in 21, 22, respectively. In the latter paper, for the sake of comparison the author has also used the differential transform method (DTM). However, only beams with polynomial forms of non-uniform cross-sectional parameters and material inhomogeneities were considered there.

Differential transformations as an approach for solving variety types of differential equations were firstly proposed by G.E. Pukhov [23] in 1976. In the beginning, the method invented by him was applied for investigation of electrical circuits [24 26] and thereafter he published a series of books [27 30], where foundations of differential transformations and their application to various pure mathematical and engineering problems were laid out and developed in detail. Recently, Bervillier [31 presented the state-of-the-art of the differential transform method in modern science, but there appear to be notable exclusions. In particular, the book of Simonyan and Avetisyan [32, where problems such as finding invariants (eigenvalue, determinant, inverse and pseudo-inverse) of nonautonomous matrices, solving linear and non-linear non-autonomous systems of finite equations have been considered and solved. Also, optimal control problems and non-autonomous matrix equations have been studied and methods of their solutions based on DTM have been proposed in [33, 34.

The differential transform method has already been applied to the vibration analysis of non-uniform cross-section and inhomogeneous beams. In [35] and [36] the authors have utilized the differential transformations to analyse free lateral vibrations of rotating tapered Euler-Bernoulli beams. Abdelghany et al. in 37. have used the DTM to compute natural frequencies and appropriate mode shapes of homogeneous beams with smooth and continuous variations of non- 
uniform cross-sections. Free vibrations of stepped FGM beams having abrupt changes of geometrical characteristics have been studied by means the DTM as well. However, it should be noticed that since cross-section and moment of inertia of the stepped beam are no longer continuous functions along the beam length in the governing differential equations, the stepped beam is analysed as an assemblage of several piecewise continuous segments and the differential transformations are applied to each of the segments accounting additionally for continuous conditions between the segments. Thus, the computational cost of the DTM increases with increasing the number of the beam sections, because more recurrent equations have to be solved. In 38 the DTM has been exploited to the free vibration analysis of FGM two-section-beams with thickwise material gradients. Natural frequencies of similar stepped FGM two-section-beams with elastically constrained ends have been calculated with the DTM in [39. In [40] and 41, the authors have proposed a differential transform element method (DTEM) improving the convergence of the DTM to examine free vibrations and stability of axially FGM tapered Euler-Bernoulli and Timoshenko beams, respectively. A vibration analysis of spinning exponentially functionally graded Timoshenko beams has been carried out with the DTM in [42.

The literature search has reveals that studies on free vibrations of axially FGM beams with varying cross-sectional parameters has been a subject of active recent research. To the best of our knowledge, DTM has not been applied to the study of free vibrations of FGM beams with arbitrary axial material gradation and cross-sectional non-uniformities varying along the beam length. Existing works are limited in the consideration to the choice of material gradients, cross-sectional parameters and boundary conditions. Having identified the gap in the literature, the objective of this paper is to present a novel approach based on the DTM for analysing free vibrations of axially functionally graded and non-uniform cross-section beams. The differential transformations will be used as a primary mathematical tool for finding natural frequencies of those beams. We transform the governing differential beam equation with arbitrary variable coefficients in connection with appropriate end supports to a set 
of recurrent algebraic equations with respect to unknown coefficients. Natural frequencies are determined from the existence condition of a non-trivial solution in the resulting system of algebraic equations. The results obtained are compared with those solutions available in the literature. In this regard, the convergence of DTM is also evaluated in the paper. A high accuracy, efficiency and versatility of the DTM is demonstrated by various examples. The examples considered are supplemented by results concerning the convergence of the DTM solutions. Finally, we analyse natural frequencies of axially FGM beams made of Aluminium Zirconia alloy $\left(\mathrm{AlZrO}_{2}\right)$ subjected to different end supports, material gradation profiles and cross-sectional parameters. The effects of the gradation parameter and the degree of non-uniformity on the natural frequencies are shown. Some computational issues being arisen in those calculations with the DTM are discussed in detail.

This paper is organize as follows. Differential transforms are introduced and their best known properties are discussed in the next section. A mathematical solution of the differential beam equation with variable coefficients arising from the free vibration of axially functionally graded beams with non-uniform crosssection along the beam length is formulated in Section 3 . Numerical results are presented and compared with existing results, where possible, in Section 4 Finally, concluding remarks are presented in Section 5

\section{A brief description of differential transformations}

Basic mathematical aspects of the DTM were presented in the original publications of the inventor of differential transformations [27-30], to which we refer for more details of this method. Throughout this paper, we use definitions and denotations introduced in those books.

Let us consider a function $A(x)$ and suppose that $A(x)$ and its all derivatives are smooth functions in a given interval $\left(x_{0}, x_{1}\right)$. The $K$ th derivative of $A(x)$ in a certain $x_{v}$ point can be considered as an $A(K)$ image (or discrete) of the original function $A(x)$. The totality of $A(K)$ images when the index goes $K=[0, \infty)$ 
will be a differential spectrum of the $A(x)$ function. Then, the basic expression to get images for a given original is the following:

$$
A(K)=\left.\frac{H^{K}}{K !} \frac{\mathrm{d}^{K} A(x)}{\mathrm{d} x^{K}}\right|_{x=x_{v}},
$$

where $A(K)$ are images of $A(x), H$ is a given constant (or so-called the scale factor), $x_{v}$ is a centre of approximation.

If a spectrum of $A(K)$ images of the original $A(x)$ is known, the original $A(x)$ may be reconstructed. This can be achieved by various ways, depending on what type of series could be used to recover the original function. In particular, using the Taylor series, the Taylor differential transformations or the T-transformations, called so by G.E. Pukhov, take the following form:

$$
A(x)=\sum_{K=0}^{\infty}\left(\frac{x-x_{v}}{H}\right)^{K} A(K) .
$$

The basic operations in the domain of differential transformations (DT) and some their properties as those in [27, 30] are listed in Table 1. It is assumed that the originals $u(x)$ and $v(x)$ have appropriate $U(K)$ and $V(K)$ differential spectra. Then, for given $U(K)$ and $V(K)$ discretes we calculate the discrete $Z(K)$, which is a differential spectrum of an $z(x)$ original. The latter is a result of algebraic manipulations between the originals $u(x)$ and $v(x)$ and differentiation of $u(x)$. It should be noted that the scale factor $H$ is taken equal to one there.

\section{DTM applied to free vibrations of inhomogeneous and non-uniform beams}

The partial differential equation that governs free vibrations of beams of length $L$, which are axially inhomogeneous and/or have a continuously variable cross-section area along the beam axis is given by [43:

$$
\frac{\partial^{2}}{\partial x^{2}}\left[D(x) \frac{\partial^{2} w}{\partial x^{2}}\right]+m(x) \frac{\partial^{2} w}{\partial t^{2}}=0,0 \leq x \leq L,
$$

where $x$ is the axial coordinate, $w$ is the transverse beam displacement, $D(x)=$ $E(x) I(x)$ is the flexural rigidity presented as a function of the axial coordinate 
Table 1: Main operations of differential transformations

\begin{tabular}{cc}
\hline Original domain & DT domain \\
\hline$z(x)=u(x)+v(x)$ & $Z(K)=U(K)+V(K)$ \\
$z(x)=c u(x)$ & $Z(K)=c U(K)$ \\
$z(x)=u(x) v(x)$ & $K \sum_{p=0}^{K} U(p) V(K-p)$ \\
$z(x)=\frac{u(x)}{v(x)}$ & $Z(K)=\frac{U(K)-\sum_{p=0}^{K} U(p) V(K-p)}{V(0)}$ \\
$z(x)=\frac{d u(x)}{d x}$ & $Z(K)=(K+1) U(K+1)$ \\
$z(x)=\frac{d^{2} u(x)}{d x^{2}}$ & $Z(K)=(K+1)(K+2) U(K+2)$ \\
$z(x)=\frac{d^{m} u(x)}{d x^{m}}$ & $Z(K)=\frac{(K+m) !}{m !} U(K+m)$ \\
$z(x)=x^{n}$ & $Z(K)=\delta(K-n)= \begin{cases}1 & K=n \\
0 & K \neq n\end{cases}$ \\
\hline
\end{tabular}

$x$ and depends on both Youngs modulus $E(x)$ and second moment of the crosssection area $I(x), m(x)=\rho(x) A(x)$ is the mass of the beam per unit length depending upon variable cross-sectional area $A(x)$ and mass density $\rho(x)$.

It is well-known that to solve (3) it is necessary to seek a temporally harmonic solution for the deflection in the form:

$$
w(x, t)=\bar{w}(x) e^{i \omega t},
$$

where $\bar{w}(x)$ is the amplitude of the beam deflection, $\omega$ is the angular frequency and $i$ is the imaginary unit. Substituting (4) into (3) reduces the initially partial differential equation (3) into an ordinary differential equation of the beam:

$$
\frac{\mathrm{d}^{2}}{\mathrm{~d} x^{2}}\left[D(x) \frac{\mathrm{d}^{2} \bar{w}(x)}{\mathrm{d} x^{2}}\right]-m(x) \omega^{2} \bar{w}(x)=0, \quad 0 \leq x \leq L .
$$

Thus, in the present study, a beam with inhomogeneous material properties and non-uniform cross-sectional parameters varying continuously along the axial direction is completely determined by the ordinary differential equation (5).

In order to find a solution of 5 using the differential transformations, it is 
convenient to rewrite it as:

$$
\frac{\mathrm{d}^{4} \bar{w}}{\mathrm{~d} x^{4}}+\bar{D}_{1}(x) \frac{\mathrm{d}^{3} \bar{w}}{\mathrm{~d} x^{3}}+\bar{D}_{2}(x) \frac{\mathrm{d}^{2} \bar{w}}{\mathrm{~d} x^{2}}-\bar{M}(x) \omega^{2} \bar{w}=0,
$$

where the variable coefficients will be denoted as $\bar{D}_{1}(x)=2 D^{\prime}(x) / D(x), \bar{D}_{2}(x)=$ $D^{\prime \prime}(x) / D(x)$ and $\bar{M}(x)=m(x) / D(x)$. Here, and in what follows, the prime denotes a derivative with respect to the $x$-coordinate.

Applying the differential transformations defined in Table 1 to Eq. (6), the latter in the differential transformation domain takes a form of a set of recurrent algebraic equations:

$$
\begin{aligned}
W(K+4) & =\frac{1}{(K+1)(K+2)(K+3)(K+4)} . \\
& {\left[\omega^{2} \sum_{p=0}^{K} W(p) M(K-p)-\right.} \\
& \sum_{p=0}^{K}(p+1)(p+2)(p+3) W(p+3) D_{1}(K-p)- \\
& \left.\sum_{p=0}^{K}(p+1)(p+2) W(p+2) D_{2}(K-p)\right]
\end{aligned}
$$

where $W(K)$ and $D_{1}(K), D_{2}(K)$ and $M(K)$ are the discretes of originals of the unknown function $\bar{w}(x)$ and the given expressions $\bar{D}_{1}(x), \bar{D}_{2}(x)$ and $\bar{M}(x)$, respectively. The last discretes are calculated using appropriate transformation rules presented in Table 1. Note that each recurrent equation of the system (7) is obtained by sequentially changing the $K$ index.

The system (7) can be reduced to a matrix equation as follows:

$$
W(K+4)=B_{K} W(0)+C_{K} W(1)+G_{K} W(2)+H_{K} W(3),
$$

where the recurrent expressions for the coefficients $B_{K}(\omega), C_{K}(\omega), G_{K}(\omega)$ and $H_{K}(\omega)$ are presented in Appendix A.

In (8), the discretes $W(0), W(1), W(2)$ and $W(3)$ are taken as unknown variables because their actual values in the differential spectrum cannot be calculated from the system of recurrent equations (7). 
If all $W(K)$ images are computed, the original function $\bar{w}(x)$ can be reconstructed according to Eq. 22 as

$$
\begin{aligned}
& \bar{w}(x, \omega)=W(0)+W(1)\left(x-x_{v}\right)+W(2)\left(x-x_{v}\right)^{2}+W(3)\left(x-x_{v}\right)^{3}+ \\
& W(4)\left(x-x_{v}\right)^{4}+\cdots+W(K)\left(x-x_{v}\right)^{K},
\end{aligned}
$$

where the discretes $W(K)$ at $K \geqslant 4$ can be expressed via the unknown discretes $W(0), W(1), W(2)$ and $W(3)$. Hence, one can get the original function in the form:

$$
\begin{aligned}
& \bar{w}(x, \omega)=W(0)+W(1)\left(x-x_{v}\right)+W(2)\left(x-x_{v}\right)^{2}+W(3)\left(x-x_{v}\right)^{3}+ \\
& {\left[B_{0} W(0)+C_{0} W(1)+G_{0} W(2)+H_{0} W(3)\right]\left(x-x_{v}\right)^{4}+} \\
& {\left[B_{1} W(0)+C_{1} W(1)+G_{1} W(2)+H_{1} W(3)\right]\left(x-x_{v}\right)^{5}+\cdots+\left[B_{K-4}+\right.} \\
& \left.W(0)+C_{K-4} W(1)+G_{K-4} W(2)+H_{K-4} W(3)\right]\left(x-x_{v}\right)^{K} .
\end{aligned}
$$

Substituting the expressions for the coefficients A.1 to A.4 into 10 and collecting common terms with respect to the unknown discretes, the original function becomes as

$$
\begin{aligned}
& \bar{w}(x, \omega)=\left(1+B_{0}\left(x-x_{v}\right)^{4}+B_{1}\left(x-x_{v}\right)^{5}+\cdots+B_{K-4}\left(x-x_{v}\right)^{K}\right) W(0)+ \\
& \left(\left(x-x_{v}\right)+C_{0}\left(x-x_{v}\right)^{4}+C_{1}\left(x-x_{v}\right)^{5}+\cdots+C_{K-4}\left(x-x_{v}\right)^{K}\right) W(1)+ \\
& \left(\left(x-x_{v}\right)^{2}+G_{0}\left(x-x_{v}\right)^{4}+G_{1}\left(x-x_{v}\right)^{5}+\cdots+G_{K-4}\left(x-x_{v}\right)^{K}\right) W(2)+ \\
& \left(\left(x-x_{v}\right)^{3}+H_{0}\left(x-x_{v}\right)^{4}+H_{1}\left(x-x_{v}\right)^{5}+\cdots+H_{K-4}\left(x-x_{v}\right)^{K}\right) W(3) .
\end{aligned}
$$

Boundary conditions imposed on the beam should be taken into account to calculate its natural frequencies. Hence, physical quantities such as rotation angle $\theta$, bending moment $M$ and shear force $Q$ involved in Eq. (3) should be defined in their explicit forms, i.e.

$$
\theta=\frac{d \bar{w}}{d x}, M=-D(x) \frac{d^{2} \bar{w}}{d x^{2}} \text { and } Q=-\frac{d}{d x}\left[D(x) \frac{d^{2} \bar{w}}{d x^{2}}\right] .
$$

In the present paper, the following end supports of the beam are considered:

- Cantilever (clamped-free) beam (C-F):

$$
\bar{w}(0)=0, \theta(0)=0, M(L)=0 \text { and } Q(L)=0 ;
$$


- Simply supported beam (S-S):

$\bar{w}(0)=0, M(0)=0, \bar{w}(L)=0$ and $M(L)=0 ;$

- Clamped-pinned beam (C-P):

$\bar{w}(0)=0, \theta(0)=0, \bar{w}(L)=0$ and $M(L)=0 ;$

- Clamped-clamped beam (C-C):

$\bar{w}(0)=0, \theta(0)=0, \bar{w}(L)=0$ and $\theta(L)=0 ;$

- Clamped-guided beam (C-G):

$\bar{w}(0)=0, \theta(0)=0, \theta(L)=0$ and $Q(L)=0$.

Using the direct differentiation of Eq. (11) with respect to the $x$ coordinate, we are able to compute any of the first three derivatives of the reconstructed original to provide the boundary conditions mentioned above. Therefore, using Eq. (8) for $\bar{w}$ in the DT domain together with appropriately transformed into the DT domain boundary conditions, which are expressed via $\bar{w}$ and its derivatives, we arrive at an eigenvalue problem for each case in the following form:

$$
\left[\begin{array}{llll}
f_{11}(\omega) & f_{12}(\omega) & f_{13}(\omega) & f_{14}(\omega) \\
f_{21}(\omega) & f_{22}(\omega) & f_{23}(\omega) & f_{24}(\omega) \\
f_{31}(\omega) & f_{32}(\omega) & f_{33}(\omega) & f_{34}(\omega) \\
f_{41}(\omega) & f_{42}(\omega) & f_{43}(\omega) & f_{44}(\omega)
\end{array}\right]\left(\begin{array}{l}
W(0) \\
W(1) \\
W(2) \\
W(3)
\end{array}\right)=0,
$$

where the functions $f_{i j}(\omega)$ are polynomials of $\omega$.

It should be noticed that the system (13) is composed of the original and its derivatives regardless boundary conditions subjected, while a discrete of the original substituted into equations of defined boundary conditions is used to formulate a similar matrix equation within a traditional application of the DTM to the eigenvalue problem, e.g. [36]. This proposed novelty will further allow us more efficiently to handle different types of constraints, and geometrical and mechanical parameters of beams without implementation of a new code for each problem. 
For the non-trivial solutions of the system $(13)$, the determinant of the matrix $\left[f_{i j}(\omega)\right]$ is set to zero, i.e.

$$
\operatorname{det}\left[f_{i j}(\omega)\right]=0 .
$$

The eigenvalue problem in (14) is a polynomial root finding task, where the $i$ th estimated eigenvalue is determined using an iterative scheme with a total number of iterations related to the accuracy of calculations. In the present work, a computational program implementing DTM for solving the free vibration problem of inhomogeneous and non-uniform beams has been developed within the Matlab environment. The eigenvalue problem is solved by the standard algorithm provided by the Matlab code with a computer using Intel ${ }^{\mathrm{TM}}$ Core $囚 \mathrm{i} 7-4770$ Quad-Core Desktop Processor 3.4 GHz with 16Gb RAM. In the calculations, the tolerance parameter between the $(n-1)$ th and $n$th iterations corresponding to four-digit precision in the calculated eigenvalues has been utilized.

It is well-known that the accuracy of solutions, obtained using the DTM, essentially depends on a number of discretes used in the Taylor series for restoring the original. The bigger number of discretes would be involved, the more accurate the approximate solution would be achieved [23]. However, in practice, the maximum numbers of discretes are always restricted by computational power and memory capacity due to quickly growing calculations needed for recurrent type equations. This fact results in increasing an error in simulations. On the other hand, the value at which the T-transformations is to be evaluated affect the accuracy of the solution as well. Therefore, to accomplish higher accuracy of the approximate solution, we will reconstruct the original (2) through the discrete spectrum found at a non-zero centre of approximation in a given solution interval. This is in contrast to the traditional approach, where a zero centre of approximation is usually used for restoring the original, e.g. [36- 42].

\section{Numerical results and discussions}

To demonstrate the general applicability and effectiveness of DTM for solving the free vibration problem for axially functionally graded and non-uniform 
Table 2: Non-dimensional natural frequencies $\Omega_{n}$ of a tapered cantilever beam with $A / A_{0}=I / I_{0}=1-0.5 x$

\begin{tabular}{|c|c|c|c|}
\hline Mode & Present & Abrate 6 ] & Huang\&Li[19] $(\mathrm{N}=10)$ \\
\hline 1 & 4.315170 & 4.315170 & 4.315170 \\
\hline 2 & 23.51926 & 23.51926 & 23.51926 \\
\hline 3 & 63.19919 & 63.19919 & 63.19919 \\
\hline 4 & 122.4377 & 122.4396 & \\
\hline
\end{tabular}

cross-section beams, we consider a variety of specific problems and compare the results obtained using the DTM with those available in the literature when possible. A general case, where there is a full arbitrariness in choosing both an axial material non-homogeneity and a cross-sectional non-uniformity simultaneously is also considered here.

\subsection{Homogeneous beams with non-uniform cross-sections}

First, a homogeneous tapered cantilever beam with cross-sectional parameters varying linearly along the beam length as functions $A / A_{0}=I / I_{0}=$ $1-0.5 x$ is considered. The first four non-dimensional natural frequencies $\Omega_{n}=\omega_{n} \sqrt{\frac{\rho A_{0} L^{4}}{E J_{0}}}$ calculated with the DTM are compared with those obtained by the Rayleigh-Ritz method in [6] and using the Fredholm integral equation in [19] are presented in Table 2. As seen from Table the present results are practically coincide with those given in the literature including higher vibration modes also.

The rate of convergence of the DTM used for calculating first four natural frequencies of the tapered cantilever beam and its dependence on the centre of approximation chosen are presented in Fig. 1. One can see that the higher frequencies require more number of discretes than lower ones to reach the exact solution. Moreover, the fastest convergence of the solution is at the central point of the given interval, while as one calculates at the zero point, the rate of convergence slows down and dramatically decelerate for the high fourth frequency. 


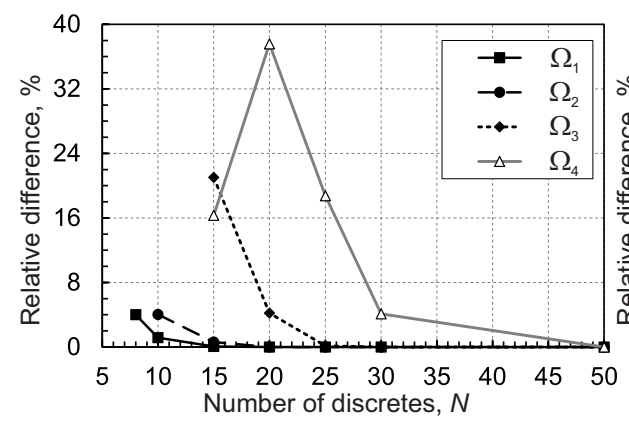

(a)

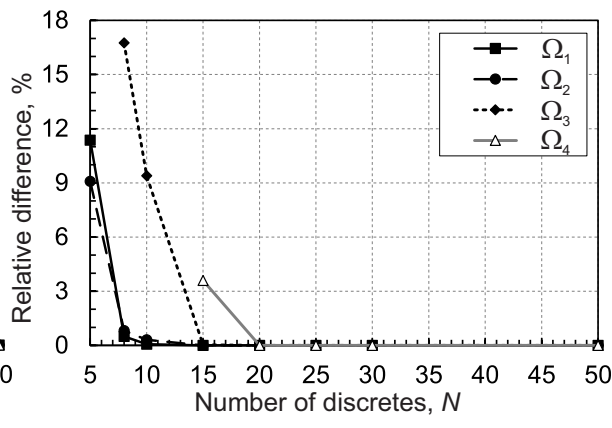

(b)

Figure 1: Convergence of the first four normalized natural frequencies of the tapered cantilever beam with $A / A_{0}=I / I_{0}=1-0.5 x$ found: (a) at $x_{v}=0$; and (b) at $x_{v}=0.5$.

This choice among the other points of the solution interval appeared the best from the standpoint of reducing the solution error. Thereby, in what follows the calculations have been carried out in the middle of given interval $x_{v}=0.5$, until another is said.

A homogeneous non-uniform beam with linearly variable cross-section area $A(x)=A_{0}(1+\alpha x)$, but with a cubic variation of the moment of inertia $I(x)=$ $I_{0}(1+\alpha x)^{3}$ is studied next. The natural frequencies of such homogeneous beam subjected to different boundary conditions have been found depending on the non-uniformity parameter $\alpha$ in [6] and [19], where the Rayleigh-Ritz method, and the Fredholm integral method and the FEM have been used, respectively. In Table 3, we present comparisons for clamped-pinned and clamped-clamped beams between the non-dimensional frequencies $\Omega_{n}$ obtained with the DTM and those results that are available in the mentioned papers.

The natural frequencies computed with the DTM coincide to those found by the other methods for the beams with the both types of boundary conditions and a variety of the non-uniformity parameter $\alpha$ used in the calculations. The dependence of the rate of convergence of the approximate fundamental frequency of the C-P beam on the non-uniformity parameter is shown in Fig. 2, It is obvious that the convergence rate of the solution practically is not sensitive 
Table 3: Non-dimensional natural frequencies $\Omega_{n}$ of beams with $A(x)=A_{0}(1+\alpha x)$ and $I(x)=I_{0}(1+\alpha x)^{3}$

\begin{tabular}{|c|c|c|c|c|c|c|}
\hline $\mathrm{BC}$ & $\alpha$ & Mode & Present & Huang\&Li [19] & Abrate [6] & FEM [19] \\
\hline \multirow[t]{18}{*}{$\mathrm{C}-\mathrm{P}$} & -0.1 & 1 & 14.848896 & 14.848896 & 14.848896 & 14.92 \\
\hline & & 2 & 47.637037 & 47.637037 & 47.637037 & \\
\hline & & 3 & 99.171635 & 99.171653 & 99.171635 & \\
\hline & 0 & 1 & 15.418206 & 15.418206 & & \\
\hline & & 2 & 49.964862 & & & \\
\hline & & 3 & 104.247696 & & & \\
\hline & 0.1 & 1 & 15.9687099 & 15.9687099 & 15.9687099 & 15.997 \\
\hline & & 2 & 52.237227 & 52.237227 & 52.237227 & \\
\hline & & 3 & 109.20235 & 109.20235 & 109.20235 & \\
\hline & 0.2 & 1 & 16.502899 & 16.502899 & 16.502899 & 16.561 \\
\hline & & 2 & 54.4614625 & 54.4614625 & 54.4614625 & \\
\hline & & 3 & 114.051623 & 114.051631 & 114.051623 & \\
\hline & 1.0 & 1 & 20.3666 & & 20.3666 & \\
\hline & & 2 & 71.04797 & & 71.04797 & \\
\hline & & 3 & 150.20086 & & 150.20086 & \\
\hline & 2.0 & 1 & 24.5826 & & 24.5826 & \\
\hline & & 2 & 89.98368 & & 89.98368 & \\
\hline & & 3 & 191.44814 & & 191.44814 & \\
\hline \multirow[t]{12}{*}{$\mathrm{C}-\mathrm{C}$} & -0.1 & 1 & 21.240978 & 21.240978 & & \\
\hline & & 2 & 58.550055 & 58.550055 & & \\
\hline & & 3 & 114.780242 & 114.780278 & & \\
\hline & 0 & 1 & 22.373285 & 22.373285 & 22.3732854 & 22.373 \\
\hline & & 2 & 61.672823 & 61.672823 & 61.672823 & \\
\hline & & 3 & 120.903392 & 120.90340 & 120.90339 & \\
\hline & 0.1 & 1 & 23.479607 & 23.479607 & 23.479607 & 23.521 \\
\hline & & 2 & 64.721068 & 64.721068 & 64.721086 & \\
\hline & & 3 & 126.87802 & 126.87805 & 126.87804 & \\
\hline & 0.2 & 1 & 24.563418 & 24.563418 & 24.563418 & 24.647 \\
\hline & & 2 & 67.704755 & 67.704755 & 67.704755 & \\
\hline & & 3 & 132.72398 & 15132.72407 & 132.72398 & \\
\hline
\end{tabular}


to small variations of the non-uniformity parameter (Fig. 2a), while it changes more clearly with increasing the parameter value (Fig. 2b). Also, it should be noticed that this tendency holds for the higher frequencies as well.

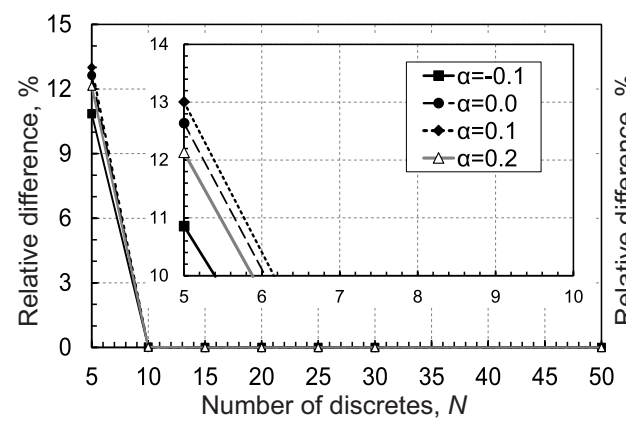

(a)

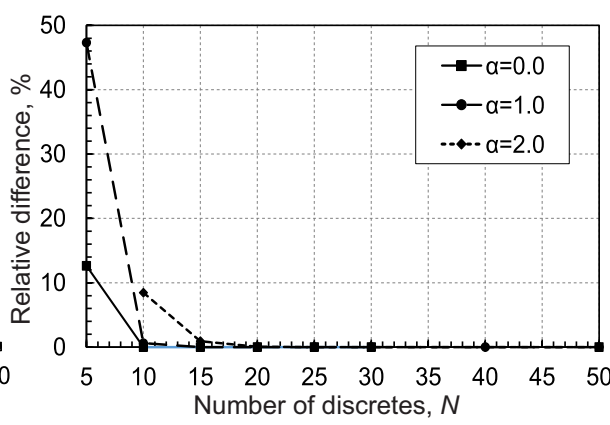

(b)

Figure 2: Convergence of the normalized fundamental frequency depending on the parameter $\alpha$ for the $\mathrm{C}-\mathrm{P}$ beam with $A(x)=A_{0}(1+\alpha x)$ and $I(x)=I_{0}(1+\alpha x)^{3}$ if: (a) $\alpha$ varies from -0.1 to 0.2 ; (b) $\alpha$ varies from 0.0 to 2.0 .

\subsection{Inhomogeneous uniform cross-section beams}

Further, we use the proposed approach implementing the DTM to compute natural frequencies of a series of uniform inhomogeneous beams, containing Young modulus $E(x)$ and mass density $\rho(x)$ that are arbitrary variable along the beam length. In the calculations we adopt there parameters in the following forms:

$$
\rho(x)=\rho_{0} \sum_{j=0}^{J} a_{j} x^{j}, E(x)=E_{0} \sum_{r=0}^{R} b_{r} x^{r},
$$

where $J$ and $R$ are any positive integers, and $a_{j}$ with $(0 \leq j \leq J)$ and $b_{r}$ with $(0 \leq r \leq R)$ are constants satisfying to requirements $\rho(x)>0, E(x)>0$ for all $x \in[0, L]$. Closed-form and approximate solutions for such inhomogeneous cantilever beam are known in [10] and [19], respectively.

Three types of beam's mass density such as: (1) constant $\rho(x) / \rho_{0}=1,(2)$ linearly changing $\rho(x) / \rho_{0}=1+x$, and (3) varying as a quadratic function $\rho(x) / \rho_{0}=1.5954+0.04 x+x^{2}$ are considered in the calculations. Each of Tables 4 to 6 represents the first five non-dimensional frequencies $k_{n}=\omega_{n}\left(\frac{\rho A_{0} L^{4}}{E J_{0}}\right)$ 
Table 4: Non-dimensional natural frequencies $k_{n}$ of the cantilever beam with $\rho(x) / \rho_{0}=1$ and $D(x) / E_{0} I=\sum_{r=0}^{R} b_{0 r} x^{r}$

\begin{tabular}{|c|c|c|c|c|c|c|}
\hline $\mathrm{R}$ & Source & $1 \mathrm{st}$ & $2 \mathrm{nd}$ & $3 \mathrm{rd}$ & 4 th & 5 th \\
\hline \multirow[t]{3}{*}{0} & Present & 17.928230 & 112.354302 & 314.595300 & 616.481229 & 1019.087644 \\
\hline & Huang\&Li [19] & 17.928232 & 112.355863 & & & \\
\hline & Weaver 43] & 17.928232 & 112.354350 & & & \\
\hline \multirow[t]{2}{*}{1} & Present & 18.899661 & 124.961973 & 355.014816 & 698.216086 & 1155.920215 \\
\hline & Huang\&Li [19] & 18.899664 & 124.961794 & & & \\
\hline \multirow[t]{2}{*}{2} & Present & 18.994321 & 127.178034 & 363.037901 & 714.808453 & 1183.929860 \\
\hline & Huang\&Li [19] & 18.994320 & 127.177553 & & & \\
\hline \multirow[t]{2}{*}{3} & Present & 18.971053 & 126.344324 & 359.531108 & 707.281060 & 1171.009309 \\
\hline & Huang\&Li [19] & 18.971054 & 126.343737 & & & \\
\hline \multirow[t]{3}{*}{4} & Present & 18.973666 & 126.471840 & 360.156611 & 708.689040 & 1173.480918 \\
\hline & Huang\&Li [19] & 18.973666 & 126.471301 & & & \\
\hline & Elishakoff[10] & 18.973666 & & & & \\
\hline
\end{tabular}

for each of the mass density and flexural rigidity relations. For the latter, appropriate coefficients can be found in Appendix B. In Tables the first two of the frequencies are compared with those available for the same cases of inhomogeneity in [10, 19] and [43]. The compared results are in very good agreement with each other for all cases of inhomogeneity adopted in the calculations.

The convergence analysis showed us that the higher is the degree of the polynomial of Young's modulus used in (15), the lower is the difference between the known results and the results obtained with DTM, and the faster the DTM is able to approximate the exact solution as seen in Fig. 3 . This conclusion is valid for all calculated frequencies regardless the degree of a polynomial of the density function. However, the higher frequencies require more number of discretes in the approximate solution than in the case of lower ones.

To demonstrate the generality of the DTM approach for modelling completely arbitrary forms of the material inhomogeneity in the vibration analysis of FGM beams, we consider the flexural rigidity and mass density as a trigono- 
Table 5: Non-dimensional natural frequencies $k_{n}$ of the cantilever beam with $\rho(x) / \rho_{0}=1+x$ and $D(x) / E_{0} I=\sum_{r=0}^{R} b_{1 r} x^{r}$

\begin{tabular}{|c|c|c|c|c|c|c|}
\hline $\mathrm{R}$ & Source & $1 \mathrm{st}$ & 2nd & 3rd & 4 th & 5 th \\
\hline \multirow[t]{2}{*}{0} & Present & 21.055898 & 140.928477 & 403.479493 & 795.279422 & 1317.894448 \\
\hline & Huang\&Li [19] & 21.055897 & 140.930444 & & & \\
\hline \multirow[t]{2}{*}{1} & Present & 22.298672 & 158.371332 & 460.631754 & 911.241917 & 1512.256542 \\
\hline & Huang\&Li [19] & 22.298670 & 158.372567 & & & \\
\hline \multirow[t]{2}{*}{2} & Present & 22.455308 & 162.368406 & 475.380847 & 941.746354 & 1563.727521 \\
\hline & Huang\&Li [19] & 22.455309 & 162.369548 & & & \\
\hline \multirow[t]{2}{*}{3} & Present & 22.452019 & 162.239233 & 474.830118 & 940.570801 & 1561.716527 \\
\hline & Huang\&Li [19] & 22.452020 & 162.240346 & & & \\
\hline \multirow[t]{2}{*}{4} & Present & 22.449333 & 162.094285 & 474.110529 & 938.967473 & 1558.918394 \\
\hline & Huang\&Li [19] & 22.449332 & 162.095281 & & & \\
\hline \multirow[t]{3}{*}{5} & Present & 22.449944 & 162.136198 & 474.350328 & 939.528605 & 1559.920652 \\
\hline & Huang\&Li [19] & 22.449944 & 162.137195 & & & \\
\hline & Elishakoff [10] & 22.449944 & & & & \\
\hline
\end{tabular}

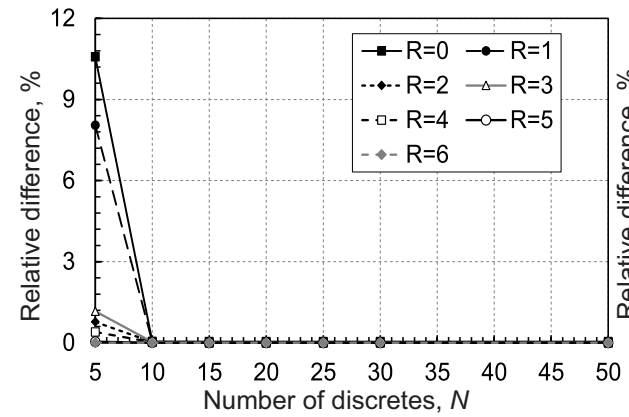

(a)

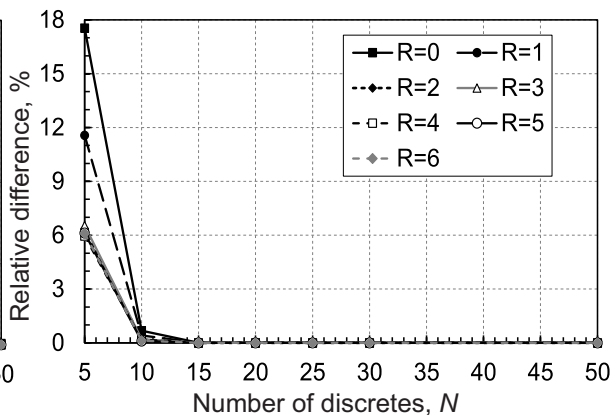

(b)

Figure 3: Convergence of normalized natural frequencies of the cantilever beam with $\rho(x) / \rho_{0}=$ $1.5954+0.04 x+x^{2}$ depending on the degree $R$ of the polynomial $D(x) / E_{0} I=\sum_{r=0}^{R} b_{2 r} x^{r}$ for: (a) the fundamental frequency; (b) the 2nd natural frequency.

metric functions of the axial coordinate in the calculations as follows:

$$
D(x)=D_{0}[1+\alpha \cos (\pi x)], \rho(x)=\rho_{0}[1+\beta \cos (\pi x)],
$$


Table 6: Non-dimensional natural frequencies $k_{n}$ of the cantilever beam with $\rho(x) / \rho_{0}=$ $1.5954+0.04 x+x^{2}$ and $D(x) / E_{0} I=\sum_{r=0}^{R} b_{2 r} x^{r}$

\begin{tabular}{ccccccc}
\hline $\mathrm{R}$ & Source & 1st & 2d & 3rd & 4 th & 5th \\
\hline 0 & Present & 24.294479 & 162.797108 & 462.716163 & 909.677354 & 1505.583556 \\
& Huang\&Li [19] & 24.294444 & 162.797574 & & & \\
1 & Present & 25.737129 & 182.906725 & 527.889460 & 1041.632735 & 1726.572091 \\
& Huang\&Li [19] & 25.737133 & 182.906151 & & & \\
2 & Present & 25.921234 & 187.554244 & 544.822636 & 1076.596904 & 1785.535421 \\
& Huang\&Li [19] & 25.921235 & 187.553379 & & & \\
3 & Present & 25.918839 & 187.461469 & 544.432450 & 1075.764990 & 1784.112810 \\
& Huang\&Li [19] & 25.918839 & 187.460556 & & & \\
4 & Present & 25.924098 & 187.739992 & 545.793135 & 1078.791133 & 1789.389455 \\
& Huang\&Li [19] & 25.924097 & 187.739261 & & & \\
5 & Present & 25.922736 & 187.648647 & 545.279363 & 1077.591252 & 1787.248519 \\
& Huang\&Li [19] & 25.922735 & 187.647888 & & & \\
6 & Present & 25.922963 & 187.666960 & 545.396356 & 1077.879518 & 1787.776220 \\
& Huang\&Li [19] & 25.922963 & 187.666166 & & & \\
& Elishakoff[10] & 25.922963 & & & & \\
\hline
\end{tabular}


where $|\alpha|<1,|\beta|<1$ are some parameters.

Tables 7 and 8 show the first six non-dimensional natural frequencies $\Omega_{n}=$ $\omega_{n} \sqrt{\frac{\rho A_{0} L^{4}}{D_{0}}}$ for the two cases of parameters $\beta=4 \alpha$ and $\beta=\alpha$, respectively, depending on the inhomogeneity parameter $\alpha$ and boundary conditions imposed.

The first tree of the frequencies computed with DTM are compared with those presented in [19, 20] for the same cases of material inhomogeneity and boundary conditions. The results match extremely well for the first two frequencies for all cases of the boundary conditions and the inhomogeneity parameter. For the third frequency the differences are moderate with the maximum distinction up to $7 \%$ in the comparisons, as seen in Fig. 4. This discrepancy results from the difference between the techniques employed for finding the approximate solutions. It is worth to notice that the DTM did not experience convergence difficulties. Though the rate of convergence depended on the boundary conditions imposed and the parameter $\alpha$ used. Moreover, the rate of convergence was slightly quicker and the computational time was somewhat lesser in the case of $\beta=\alpha$ than those in the case of $\beta=4 \alpha$. It means that increasing in-

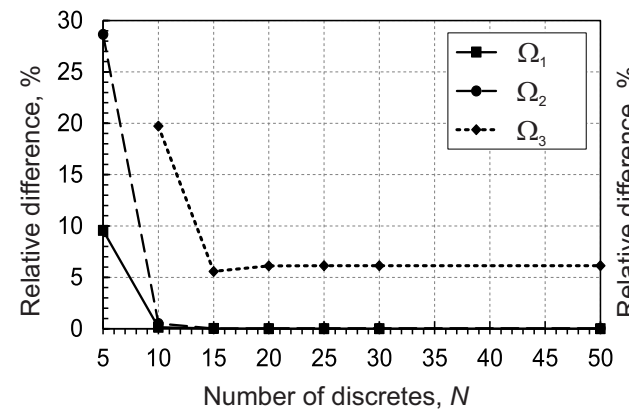

(a)

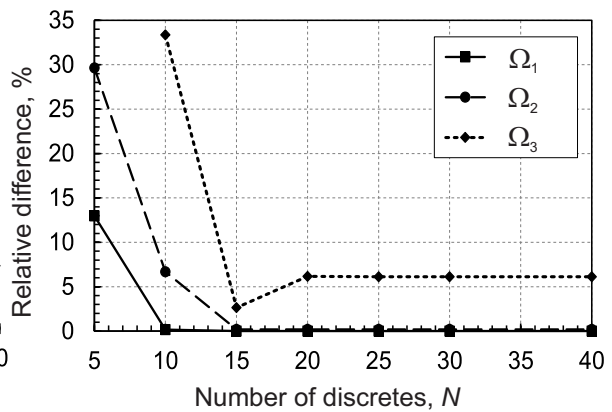

(b)

Figure 4: Convergence of approximate solutions of normalized natural frequencies of the $\mathrm{C}$ - C beam with $D(x)=D_{0}[1+\alpha \cos (\pi x)]$ and $\rho(x)=\rho_{0}[1+\beta \cos (\pi x)]$, and $\alpha=0.2$ if: (a) $\beta=\alpha$; (b) $\beta=4 \alpha$.

homogeneity of the mechanical parameter along the beam length increases the computational cost of the DTM needed for restoring an exact solution. Also we revealed that unlike the method used in [19] all natural frequencies obtained 
Table 7: Non-dimensional natural frequencies $\Omega_{n}$ of beams with $D(x)=D_{0}[1+\alpha \cos (\pi x)]$ and $\rho(x)=\rho_{0}[1+\beta \cos (\pi x)]$, if $\beta=4 \alpha$

\begin{tabular}{|c|c|c|c|c|c|c|c|c|c|}
\hline \multirow[t]{2}{*}{$\mathrm{BC}$} & \multirow[t]{2}{*}{$\mathrm{n}$} & \multirow[t]{2}{*}{ Source } & \multicolumn{7}{|c|}{$\alpha$} \\
\hline & & & -0.2 & -0.15 & -0.1 & 0 & 0.1 & 0.15 & 0.2 \\
\hline \multirow[t]{9}{*}{$C-F$} & 1 & Present & 2.56899 & 2.75721 & 2.97143 & 3.51602 & 4.34283 & 4.96987 & 5.89084 \\
\hline & & Huang\&Li [19] & 2.5690 & 2.7572 & 2.9714 & 3.5160 & 4.3428 & 4.9699 & 5.8908 \\
\hline & 2 & Present & 20.54575 & 20.84940 & 21.18732 & 22.03449 & 23.40750 & 24.64429 & 27.05785 \\
\hline & & Huang\&Li [19] & 20.5462 & 20.8498 & 21.1877 & 22.0345 & 23.4080 & 24.6452 & 27.0597 \\
\hline & 3 & Present & 64.03571 & 62.80216 & 61.99739 & 61.69721 & 63.47331 & 65.61956 & 69.88894 \\
\hline & & Huang\&Li 19] & 64.1287 & 62.8517 & 62.0246 & 61.7151 & 63.5303 & 65.7357 & 70.1756 \\
\hline & 4 & Present & 128.86866 & 124.88568 & 122.43363 & 120.90192 & 123.54569 & 127.15568 & 134.15760 \\
\hline & 5 & Present & 215.28680 & 207.44420 & 202.86979 & 199.85950 & 203.75458 & 209.32144 & 220.05414 \\
\hline & 6 & Present & 324.69770 & 311.43252 & 303.79480 & 298.55314 & 303.94195 & 312.28903 & 333.53432 \\
\hline \multirow[t]{9}{*}{$S-S$} & 1 & Present & 9.86960 & 9.86960 & 9.86960 & 9.86960 & 9.86960 & 9.86960 & 9.86960 \\
\hline & & Huang\&Li [19] & 9.8696 & 9.8696 & 9.8696 & 9.8696 & 9.8696 & 9.8696 & 9.8696 \\
\hline & 2 & Present & 42.53832 & 41.13895 & 40.19695 & 39.47842 & 40.19695 & 41.13895 & 42.53832 \\
\hline & & Huang\&Li [19] & 42.5405 & 41.1404 & 40.1979 & 39.4791 & 40.1979 & 41.1404 & 42.5522 \\
\hline & 3 & Present & 96.74878 & 92.75051 & 90.42512 & 88.82644 & 90.42512 & 92.7505 & 96.74878 \\
\hline & & Huang\&Li 19] & 98.9439 & 92.4350 & 90.4469 & 88.8481 & 90.3370 & 92.4350 & 98.6659 \\
\hline & 4 & Present & 172.31388 & 164.80929 & 160.69538 & 157.91367 & 160.69538 & 164.80929 & 172.31388 \\
\hline & 5 & Present & 269.24338 & 257.39772 & 251.03594 & 246.74011 & 251.03594 & 257.39772 & 269.24338 \\
\hline & 6 & Present & 387.46288 & 370.51003 & 361.43981 & 355.30580 & 361.43981 & 370.51003 & 387.46288 \\
\hline \multirow[t]{9}{*}{$\mathrm{C}-\mathrm{P}$} & 1 & Present & 14.21170 & 14.49167 & 14.78504 & 15.41821 & 16.12346 & 16.50640 & 16.91055 \\
\hline & & Huang\&Li [19] & 14.2117 & 14.4917 & 14.7850 & 15.4182 & 16.1235 & 16.5065 & 16.9107 \\
\hline & 2 & Present & 51.51352 & 50.72796 & 50.19312 & 49.96486 & 51.14342 & 52.46453 & 54.46396 \\
\hline & & Huang\&Li [19] & 51.5819 & 50.7722 & 50.2210 & 49.9742 & 51.1459 & 52.4695 & 54.4812 \\
\hline & 3 & Present & 110.84949 & 107.62986 & 105.59270 & 104.24770 & 106.29373 & 109.07530 & 113.90842 \\
\hline & & Huang\&Li 19] & 112.9319 & 110.0300 & 108.2707 & 107.4485 & 110.4157 & 113.7307 & 119.2732 \\
\hline & 4 & Present & 191.82757 & 185.02266 & 180.99163 & 178.26973 & 181.53790 & 186.20726 & 194.74699 \\
\hline & 5 & Present & 294.30289 & 282.91292 & 276.42480 & 272.03097 & 276.87027 & 283.89975 & 296.98341 \\
\hline & 6 & Present & 418.41898 & 401.33012 & 391.90015 & 385.53145 & 392.27584 & 402.09670 & 420.24635 \\
\hline \multirow[t]{9}{*}{$C-C$} & 1 & Present & 22.36955 & 22.37119 & 22.37235 & 22.37329 & 22.37235 & 22.37119 & 22.36955 \\
\hline & & Huang\&Li 19] & 22.3700 & 22.3715 & 22.3726 & 22.3735 & 22.3726 & 22.3715 & 22.3700 \\
\hline & 2 & Present & 64.64605 & 63.32288 & 62.39804 & 61.67282 & 62.39804 & 63.32288 & 64.64605 \\
\hline & & Huang\&Li [9] & 64.7658 & 63.3937 & 62.4327 & 61.6883 & 62.4330 & 63.3897 & 64.7668 \\
\hline & 3 & Present & 129.32930 & 125.27614 & 122.73650 & 120.90339 & 122.73650 & 125.27614 & 129.32930 \\
\hline & & Huang\&Li [19] & 138.6441 & 132.6284 & 131.3240 & 129.2174 & 131.2343 & 132.3560 & 137.759 \\
\hline & 4 & Present & 215.63076 & 207.74116 & 203.10268 & 199.85945 & 203.10268 & 207.74116 & 215.63076 \\
\hline & 5 & Present & 323.43250 & 310.72825 & 303.52343 & 298.55554 & 303.52343 & 310.72825 & 323.43250 \\
\hline & 6 & Present & 452.43908 & 434.13230 & 423.96477 & 416.99101 & 423.96477 & 434.13230 & 452.43908 \\
\hline
\end{tabular}


Table 8: Non-dimensional natural frequencies $\Omega_{n}$ of beams with $D(x)=D_{0}[1+\alpha \cos (\pi x)]$ and $\rho(x)=\rho_{0}[1+\beta \cos (\pi x)]$, if $\beta=\alpha$

\begin{tabular}{|c|c|c|c|c|c|c|c|c|c|}
\hline \multirow[t]{2}{*}{$\mathrm{BC}$} & \multirow[t]{2}{*}{$\mathrm{n}$} & \multirow[t]{2}{*}{ Source } & \multicolumn{7}{|c|}{$\alpha$} \\
\hline & & & -0.2 & -0.15 & -0.1 & 0 & 0.1 & 0.15 & 0.2 \\
\hline \multirow[t]{9}{*}{$C-F$} & 1 & Present & 3.02240 & 3.14158 & 3.26323 & 3.51602 & 3.78526 & 3.92769 & 4.07635 \\
\hline & & Huang\&Li 19] & 3.0024 & 3.1416 & 3.2632 & 3.5160 & 3.7853 & 3.9277 & 4.0763 \\
\hline & 2 & Present & 21.20726 & 21.41809 & 21.62542 & 22.03449 & 22.44457 & 22.65349 & 22.86721 \\
\hline & & Huang\&Li 19] & 21.2069 & 21.4179 & 21.6255 & 22.0345 & 22.4447 & 22.6534 & 22.8668 \\
\hline & 3 & Present & 61.22461 & 61.33987 & 61.45613 & 61.69721 & 61.95882 & 62.10089 & 62.25261 \\
\hline & & Huang\&Li 19] & 61.2666 & 61.3741 & 61.4838 & 61.7151 & 61.9758 & 62.1192 & 62.2737 \\
\hline & 4 & Present & 120.57744 & 120.65392 & 120.73270 & 120.90192 & 121.09441 & 121.20274 & 121.32110 \\
\hline & 5 & Present & 199.61965 & 199.67285 & 199.73064 & 199.85953 & 200.01244 & 200.10102 & 200.19772 \\
\hline & 6 & Present & 298.35875 & 298.40832 & 298.45270 & 298.55552 & 298.68294 & 298.76147 & 298.83443 \\
\hline \multirow[t]{9}{*}{$S-S$} & 1 & Present & 9.83953 & 9.85280 & 9.86217 & 9.86960 & 9.86217 & 9.85280 & 9.83953 \\
\hline & & Huang\&Li 19] & 9.8395 & 9.8528 & 9.8622 & 9.8696 & 9.8622 & 9.8528 & 9.8395 \\
\hline & 2 & Present & 39.52073 & 39.50227 & 39.48903 & 39.47842 & 39.48903 & 39.50227 & 39.52073 \\
\hline & & Huang\&Li 19] & 39.5239 & 39.4045 & 39.4905 & 39.4791 & 39.4905 & 39.4045 & 39.5239 \\
\hline & 3 & Present & 88.87424 & 88.85299 & 88.83814 & 88.82644 & 88.83814 & 88.85299 & 88.87424 \\
\hline & & Huang\&Li 19] & 90.2491 & 90.2874 & 90.3149 & 88.8481 & 90.3149 & 90.2874 & 90.2491 \\
\hline & 4 & Present & 157.96291 & 157.94097 & 157.92570 & 157.91367 & 157.92570 & 157.94097 & 157.96291 \\
\hline & 5 & Present & 246.79126 & 246.76778 & 246.75224 & 246.74011 & 246.75224 & 246.76778 & 246.79126 \\
\hline & 6 & Present & 355.36156 & 355.33150 & 355.31812 & 355.30580 & 355.31812 & 355.33150 & 355.36156 \\
\hline \multirow[t]{9}{*}{$\mathrm{C}-\mathrm{P}$} & 1 & Present & 14.91970 & 15.05273 & 15.17990 & 15.41821 & 15.63677 & 15.73901 & 15.83660 \\
\hline & & Huang\&Li [19] & 14.9196 & 15.0527 & 15.1799 & 15.4182 & 15.6367 & 15.7389 & 15.8365 \\
\hline & 2 & Present & 49.67240 & 49.74997 & 49.82387 & 49.96486 & 50.10256 & 50.17204 & 50.24281 \\
\hline & & Huang\&Li [19] & 49.6719 & 49.7506 & 49.8265 & 49.9742 & 50.1206 & 50.1944 & 50.2691 \\
\hline & 3 & Present & 104.05672 & 104.10517 & 104.15266 & 104.24770 & 104.34732 & 104.40054 & 104.45702 \\
\hline & & Huang\&Li 19] & 107.5159 & 107.5407 & 107.5357 & 107.4485 & 107.2753 & 107.1613 & 107.0311 \\
\hline & 4 & Present & 178.13492 & 178.16704 & 178.19982 & 178.26973 & 178.34898 & 178.39352 & 178.44241 \\
\hline & 5 & Present & 271.93307 & 271.95304 & 271.97670 & 272.03097 & 272.09750 & 272.13663 & 272.18249 \\
\hline & 6 & Present & 385.44723 & 385.47001 & 385.48867 & 385.53145 & 385.58880 & 385.62228 & 385.68087 \\
\hline \multirow[t]{9}{*}{$C-C$} & 1 & Present & 22.29882 & 22.33172 & 22.35491 & 22.37329 & 22.35491 & 22.33172 & 22.29882 \\
\hline & & Huang\&Li [19 & 22.2984 & 22.3316 & 22.3549 & 22.3735 & 22.3549 & 22.3316 & 22.2984 \\
\hline & 2 & Present & 61.62357 & 61.64563 & 61.66089 & 61.67282 & 61.66089 & 61.64563 & 61.62357 \\
\hline & & Huang\&Li 19] & 61.6542 & 61.6699 & 61.6804 & 61.6883 & 61.6804 & 61.6699 & 61.6542 \\
\hline & 3 & Present & 120.87930 & 120.89014 & 120.89759 & 120.90339 & 120.89759 & 120.89014 & 120.87930 \\
\hline & & Huang\&Li [19] & 128.7765 & 128.9739 & 129.1098 & 129.2174 & 129.1098 & 128.9739 & 128.7780 \\
\hline & 4 & Present & 199.85145 & 199.85512 & 199.85759 & 199.85945 & 199.85759 & 199.85512 & 199.85145 \\
\hline & 5 & Present & 298.55941 & 298.55688 & 298.55625 & 298.55554 & 298.55625 & 298.55688 & 298.55941 \\
\hline & 6 & Present & 417.00069 & 416.99397 & 416.99399 & 416.99101 & 416.99399 & 416.99397 & 417.00069 \\
\hline
\end{tabular}


using the DTM are exactly even functions of the parameter $\alpha$ in the case of the symmetric boundary conditions S-S and C-C, while in the compared method, the results are symmetrical with respect to the sign of $\alpha$ only if $\beta=\alpha$.

\subsection{Axially functionally graded beams with uniform and non-uniform cross- sections}

We illustrate now an application of DTM to the free vibration analysis of beams made of functionally graded materials with any sophisticated axial gradation profiles. At the beginning, let us consider a beam with uniform cross section area $A_{0}$ and second moment of area $I_{0}$, but with variable Young modulus $E(x)$ and mass density $\rho(x)$ as the following functions of the $x$-coordinate:

$$
\begin{cases}E(x)=E_{1}\left(1-\frac{e^{\alpha x}-1}{e^{\alpha}-1}\right)+E_{2} \frac{e^{\alpha x}-1}{e^{\alpha}-1}, & \alpha \neq 0 \\ E(x)=E_{1}(1-x)+E_{2} x, & \alpha=0\end{cases}
$$

and

$$
\begin{cases}\rho(x)=\rho_{1}\left(1-\frac{e^{\alpha x}-1}{e^{\alpha}-1}\right)+\rho_{2} \frac{e^{\alpha x}-1}{e^{\alpha}-1}, & \alpha \neq 0 \\ \rho(x)=\rho_{1}(1-x)+\rho_{2} x, & \alpha=0,\end{cases}
$$

where $E_{1}, \rho_{1}$ and $E_{2}, \rho_{2}$ are corresponding Young modulus and mass density of material at the ends $x=0$ and $x=L$, respectively, and $\alpha$ is the gradation parameter describing the volume fraction change along the beam length.

For the sake of comparison, in the calculations we accept functionally graded metal/ceramic Aluminium Zirconia alloy $\left(\mathrm{AlZrO}_{2}\right)$, the material properties of which are presented in [19] and are given as follows:

$$
\begin{array}{rllll}
\mathrm{Al}: & E_{a}=70 \quad \mathrm{GPa}, & \rho_{a}=2702 & \mathrm{~kg} / \mathrm{m}^{3} \\
\mathrm{ZrO}_{2}: & E_{z}=200 & \mathrm{GPa}, & \rho_{z}=5700 & \mathrm{~kg} / \mathrm{m}^{3}
\end{array}
$$

The results of the free vibration analysis of axially functionally graded beams with two types of gradation profiles, when the metal phase $\mathrm{Al}$ is rich near the end $x=0$ and the ceramic phase $\mathrm{ZrO}_{2}$ is rich near the end $x=L$ (case 1 ), and the metal phase $\mathrm{Al}$ is rich near the end $x=L$ and the ceramic phase $\mathrm{ZrO}_{2}$ is 
Table 9: Non-dimensional fundamental frequencies $\Omega_{n}$ of axially graded $\mathrm{AlZrO}_{2}$ beams

\begin{tabular}{|c|c|c|c|c|c|c|c|c|c|}
\hline \multirow[t]{2}{*}{$\alpha$} & \multirow[b]{2}{*}{ Source } & \multicolumn{2}{|c|}{$\mathrm{C}-\mathrm{F}$} & \multicolumn{2}{|c|}{$S-S$} & \multicolumn{2}{|c|}{ C - P } & \multicolumn{2}{|c|}{$\mathrm{C}-\mathrm{C}$} \\
\hline & & Case 1 & Case 2 & Case 1 & Case 2 & Case 1 & Case 2 & Case 1 & Case 2 \\
\hline \multirow[t]{4}{*}{-10} & Present & 3.47486 & 4.05056 & 11.46179 & 9.93313 & 16.40307 & 17.31412 & 24.06385 & 24.79734 \\
\hline & Huang\&Li [19] & 3.5656 & 4.1800 & 11.4532 & 9.9358 & 16.4775 & 17.2993 & 24.0576 & 24.7949 \\
\hline & Hein\&Feklistova 20] & & & 11.4481 & & 16.3837 & & 24.0269 & \\
\hline & Error, \% & 2.545 & 3.097 & 0.095 & 0.027 & 0.452 & 0.086 & 0.0260 & 0.056 \\
\hline \multirow[t]{4}{*}{-3} & Present & 3.14088 & 4.83168 & 11.24428 & 10.36686 & 16.02546 & 17.86998 & 23.94328 & 24.93636 \\
\hline & Huang\&Li 19] & 3.1421 & 4.8317 & 11.2443 & 10.3669 & 16.0307 & 17.8701 & 23.9456 & 24.9375 \\
\hline & Hein\&Feklistova 20] & & & 11.2422 & & 16.0307 & & 23.9384 & \\
\hline & Error, \% & 0.0401 & 0.0004 & 0.0002 & 0.0004 & 0.0326 & 0.0007 & 0.0097 & 0.0046 \\
\hline \multirow[t]{4}{*}{0} & Present & 2.92561 & 5.01564 & 10.86634 & 10.86634 & 15.87338 & 17.91475 & 24.37535 & 24.37535 \\
\hline & Huang\&Li 19] & 2.9256 & 5.0156 & 10.8663 & 10.8663 & 15.8734 & 17.9147 & 24.3752 & 24.3752 \\
\hline & Hein\&Feklistova 20] & & & 10.8660 & & 15.8729 & & 24.3749 & \\
\hline & Error, \% & 0.0003 & 0.0008 & 0.0004 & 0.0004 & 0.0001 & 0.0003 & 0.0006 & 0.0138 \\
\hline \multirow[t]{4}{*}{3} & Present & 2.85446 & 4.84663 & 10.36686 & 11.24428 & 15.71686 & 17.88728 & 24.93636 & 23.94329 \\
\hline & Huang\&Li 19 & 2.8544 & 4.8466 & 10.3669 & 11.2443 & 15.7171 & 17.8867 & 24.9375 & 23.9456 \\
\hline & Hein\&Feklistova 20] & & & 10.3670 & & 15.7171 & & 24.9371 & \\
\hline & Error, \% & 0.0021 & 0.0006 & 0.0286 & 0.0002 & 0.0015 & 0.0032 & 0.0046 & 0.0097 \\
\hline \multirow[t]{4}{*}{10} & Present & 3.09642 & 4.46502 & 9.93277 & 11.4582 & 15.47935 & 17.91852 & 24.81462 & 24.05657 \\
\hline & Huang\&Li 19] & 3.0431 & 4.4629 & 9.9358 & 11.4532 & 15.4930 & 17.9050 & 24.7949 & 24.0576 \\
\hline & Hein\&Feklistova 20] & & & 9.9366 & & 15.4930 & & 24.8080 & \\
\hline & Error, \% & 1.752 & 0.048 & 0.031 & 0.044 & 0.088 & 0.076 & 0.080 & 0.004 \\
\hline $\mathrm{HB}^{1}$ & & 3.5 & 160 & & & 15.4 & 182 & & 733 \\
\hline
\end{tabular}

${ }^{1} H B$ is a homogeneous beam

rich near the end $x=0$ (case 2), subjected to different boundary conditions are collected in Tables 9 and 10. Table 9 demonstrates non-dimensional fundamental frequencies $\Omega_{n}=\omega_{n} \sqrt{\frac{\rho_{a} A_{0} L^{4}}{E_{a} I_{0}}}$ of the beams compared the present results with those available in the literature. Table 10 shows the other first five nondimensional frequencies $\Omega_{n}$ depending on the gradation parameter $\alpha$ and the boundary conditions.

One can see in Table 9 that the frequencies calculated with DTM are mainly in a good agreement with those obtained by the other methods in [19, 20]. The maximum relative differences between the results vary from $1.7 \%$ to $3 \%$ in the case of the cantilever beam with the highest material parameter $\alpha= \pm 10$, but they are hundredths and thousandths of a percent for the smaller values of this parameter and more restrained boundary conditions.

The convergence analysis of these solutions with respect to the gradation parameter $\alpha$ and boundary conditions has been performed and revealed some 
computational issues. It was found out that in the case of $\alpha=-10$, the approximate solution of the FGM beam with the loosest C-F constraints for the both gradation patterns case 1 and case 2 exhibits oscillations and grows rapidly with slow increasing $N$, when the number of discretes surpasses a certain threshold as shown in Fig. 5 a. This limit value was equal to $N=53$ in the calculations. The DTM solutions for that beam under more restrained boundary conditions than the C-F constraints do not show a strong oscillatory behaviour, but they feature a divergence phenomenon after $N=53$ as seen in Fig. 6 6 . In the presence of

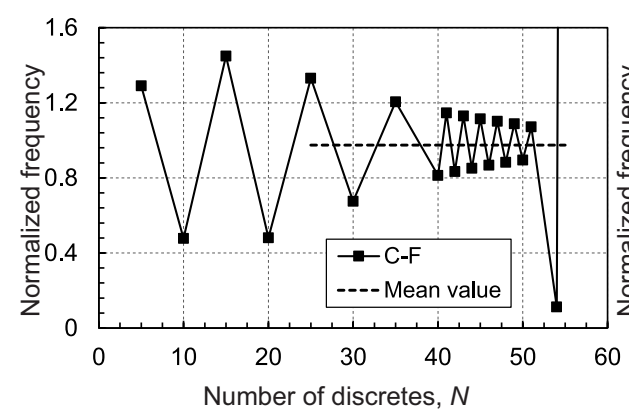

(a)

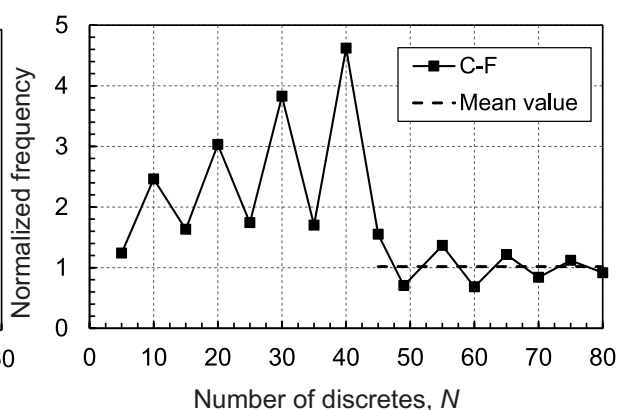

(b)

Figure 5: Convergence of the fundamental frequency normalized with respect to the solution in [19], $\frac{\Omega_{1}}{\Omega_{1}^{*}}$ for the C-F beam in the case 1: (a) $\alpha=-10$; and (b) $\alpha=10$.

such computational issues the accuracy of the DTM deteriorates. This is due to the well known phenomenon for the higher order polynomial terms which could lead to round-off errors and ill-conditioning for finding the roots of a polynomial in the eigenvalue problem [44. This computational instability cannot be merely overcome by increasing a number of discretes. Perhaps, a multistep approach within the DTM [22, 27, 40] is more suitable for solving problems involving high material gradients in FGM structures. Nevertheless, one can see in Table 10 that the frequencies averaged over a range, where spurious oscillations occur, are of a good compliance with the results in the literature, and the differential transform of 50 discretes is an reasonable quantity to achieve a demand for high accuracy of the solutions as well. 
In the case of $\alpha=10$, the DTM solutions are less sensitive to rounding errors. The approximate solution of the C-F beam oscillates near an averaged value and tends to it with increasing $N$, Fig. 5 b. The approximate solutions for beams subjected to the other boundary conditions are non-oscillatory and show the convergence, but the rate of convergence slows down remarkably in compared with the examples previously mentioned in the paper as illustrated in Fig. 6b. In the cases of $\alpha=0$ and \pm 3 , the convergence of DTM solutions

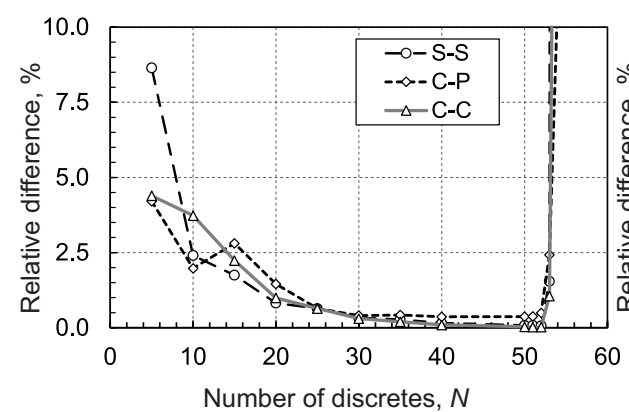

(a)

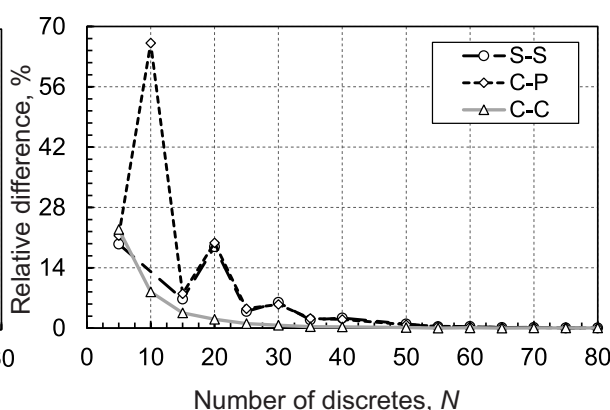

(b)

Figure 6: Convergence of the fundamental frequencies normalized with respect to the solutions in [19] for the beams under S-S, C-P and C-C constraints in the case 1: (a) $\alpha=-10$; and (b) $\alpha=10$.

does not demonstrate any computational issues and possesses a fast rate of convergence regardless boundary conditions applied to the FGM beam.

The results listed in Table 10 present higher mode frequencies of the considered FGM beam. It is worth to notice that the DTM solutions of the higher frequencies have the same convergence behaviour as those described above for the fundamental frequency. Therefore, in the cases of C-F beam with $\alpha= \pm 10$ the averaging and truncation techniques mentioned above were applied for recovering the results. As seen in Table, the higher frequencies of the beams under symmetric boundary conditions in the case 1 are identical to ones in the case 2 for the equal, but opposite sign inhomogeneity parameter $\alpha$. Moreover, the calculated frequencies indicate a complex variation tendency depending on that parameter. This fact proves a strong dependence of free vibrations on FGM 
Table 10: Non-dimensional natural frequencies $\Omega_{n}$ of axially graded $\mathrm{AlZrO}_{2}$ beams

\begin{tabular}{|c|c|c|c|c|c|c|c|c|c|}
\hline \multirow[t]{2}{*}{$\alpha$} & \multirow[b]{2}{*}{ Mode } & \multicolumn{2}{|c|}{$C-F$} & \multicolumn{2}{|c|}{$S-S$} & \multicolumn{2}{|c|}{$C-P$} & \multicolumn{2}{|c|}{$C-C$} \\
\hline & & Case 1 & Case 2 & Case 1 & Case 2 & Case 1 & Case 2 & Case 1 & Case 2 \\
\hline \multirow[t]{5}{*}{-10} & 2 & 23.75440 & 24.29463 & 45.70803 & 40.01590 & 54.92971 & 54.01091 & 68.11671 & 66.33548 \\
\hline & 3 & 68.13491 & 66.30623 & 102.72185 & 90.70289 & 116.42524 & 111.04684 & 135.35756 & 128.25552 \\
\hline & 4 & 135.43341 & 128.78960 & 182.41317 & 160.67124 & 200.98782 & 186.87381 & 225.52083 & 210.67467 \\
\hline & 5 & 225.49512 & 210.07984 & 284.85677 & 254.31263 & 308.11954 & 290.92369 & 338.63110 & 311.36029 \\
\hline & 6 & 338.79814 & 334.51364 & 410.13045 & 354.47104 & 438.49036 & 409.84991 & 474.60218 & 448.14160 \\
\hline \multirow[t]{5}{*}{-3} & 2 & 23.05185 & 25.49215 & 44.85368 & 41.96811 & 54.46761 & 54.92984 & 67.75525 & 67.10406 \\
\hline & 3 & 67.47564 & 67.78661 & 100.81154 & 94.50370 & 115.51745 & 112.79363 & 134.43877 & 130.22820 \\
\hline & 4 & 134.15354 & 130.83415 & 179.10915 & 167.99936 & 199.05597 & 191.57750 & 223.57897 & 214.26212 \\
\hline & 5 & 223.31328 & 214.83999 & 279.74462 & 262.46802 & 305.02380 & 291.31830 & 335.14367 & 319.25327 \\
\hline & 6 & 334.88852 & 319.81271 & 402.71983 & 377.91596 & 433.39133 & 412.03012 & 469.10471 & 445.21482 \\
\hline \multirow[t]{5}{*}{0} & 2 & 22.35382 & 26.46480 & 43.66381 & 43.66381 & 53.95855 & 55.97100 & 67.58746 & 67.58746 \\
\hline & 3 & 66.16920 & 70.26689 & 98.11772 & 98.11772 & 113.77010 & 115.83950 & 132.83907 & 132.83907 \\
\hline & 4 & 131.42546 & 135.61105 & 174.30881 & 174.30881 & 195.35569 & 197.45609 & 219.85463 & 219.85463 \\
\hline & 5 & 218.46231 & 222.69830 & 272.24998 & 272.24998 & 298.70768 & 300.82891 & 328.64103 & 328.64103 \\
\hline & 6 & 327.26046 & 331.53089 & 391.94625 & 391.94625 & 423.82371 & 425.95976 & 459.19322 & 459.19322 \\
\hline \multirow[t]{5}{*}{3} & 2 & 21.49489 & 27.14143 & 41.96811 & 44.85372 & 52.80201 & 56.89965 & 67.10406 & 67.75527 \\
\hline & 3 & 63.67157 & 72.77856 & 94.50370 & 100.81159 & 110.60535 & 118.43481 & 130.22820 & 134.43881 \\
\hline & 4 & 126.57717 & 140.33777 & 167.99936 & 179.10929 & 189.36052 & 202.30712 & 214.26212 & 223.57908 \\
\hline & 5 & 210.50632 & 230.10699 & 262.46802 & 279.74473 & 289.08361 & 308.52179 & 319.25327 & 335.14381 \\
\hline & 6 & 315.42943 & 342.13279 & 377.91596 & 402.72007 & 409.78356 & 437.07867 & 445.21482 & 469.10516 \\
\hline \multirow[t]{5}{*}{10} & 2 & 21.06942 & 27.07263 & 39.98589 & 45.69440 & 50.75565 & 57.79663 & 66.26926 & 68.09948 \\
\hline & 3 & 60.59987 & 74.40182 & 90.72143 & 102.66569 & 106.34852 & 120.46246 & 127.89884 & 135.31837 \\
\hline & 4 & 123.75115 & 144.11101 & 160.71032 & 182.38506 & 182.12175 & 205.87632 & 209.61761 & 225.46878 \\
\hline & 5 & 208.28604 & 236.40866 & 254.25563 & 284.88178 & 278.30040 & 314.05743 & 311.40310 & 338.61609 \\
\hline & 6 & 332.59071 & 351.30339 & 354.46540 & 410.12223 & 386.07054 & 444.99713 & 447.88063 & 474.45889 \\
\hline
\end{tabular}


material parameters and the importance of their predictions at the design stage.

Finally, we consider functionally graded beams with axially graded material properties defined by the relations 17 and 18 for the two gradation patterns (case 1) and (case 2) mentioned in the previous simulations with nonuniform cross-sectional parameters. The beams are assumed to have a variable cross-section given by functions of $x$ as follows: $A(x)=A_{0}(1+\beta x)^{2}$ and $I(x)=I_{0}(1+\beta x)^{4}$. These geometry relations may correspond to, for instance, a beam with a rectangular cross-section whose height and width vary linearly with the same taper ratio or a beam with a circular cross-section and linearly tapering diameter. Tables 11 to 14 contain non-dimensional natural frequencies $\Omega_{n}=\omega_{n} \sqrt{\frac{\rho_{a} A L^{4}}{E_{a} I}}$ computed depending on values of inhomogeneity $\alpha$ and non-uniformity $\beta$ parameters at different boundary conditions.

The convergence analysis has been performed for each case study. Analogously to the previous calculations, the oscillations and divergence behaviour of the DTM solutions in the regions of high material gradients $\alpha= \pm 10$ occur. Herewith, these phenomena become stronger with extending the cross-sectional taper towards the free end of C-F beam as seen in Fig. 7. To recover the natural frequencies from the results suffered from the computational instabilities, the averaging and truncation techniques mentioned above have been used in the post-processing stage. In the other examples, where computational issues did not arise, we have observed only a slight slowing down the convergence rate with increasing the taper ratio towards unity, for instance, in the case of C-C beam as shown in Fig. 8. The DTM solutions of frequencies of beams with low material gradients $\alpha=0$ and $\alpha= \pm 3$ have had no any computational difficulties and have featured fast enough convergence rate and relatively low computational cost at the variety of taper ratios and boundary conditions.

The results in Tables 11 to 14 indicate that for axially functionally graded beams, the natural frequencies show a complex variation behaviour with respect to the both material inhomogeneity and geometrical non-uniformity and they differ significantly from beams having only material inhomogeneity or only nonuniform cross-section at the same boundary conditions. In doing so, the higher 
Table 11: Non-dimensional natural frequencies $\Omega_{n}$ of an axially functionally graded and nonuniform cross-section cantilever beam with $A(x)=(1+\beta x)^{2}$ and $I(x)=(1+\beta x)^{4}$, and $E(x)$ and $\rho(x)$ changing in accordance with 17 and 18

\begin{tabular}{|c|c|c|c|c|c|c|c|c|c|c|c|c|}
\hline & \multirow{3}{*}{$\beta$} & \multirow{3}{*}{ Mode } & \multicolumn{10}{|c|}{$\alpha$} \\
\hline & & & \multicolumn{2}{|c|}{-10} & \multicolumn{2}{|c|}{-3} & \multicolumn{2}{|c|}{0} & \multicolumn{2}{|c|}{3} & \multicolumn{2}{|c|}{10} \\
\hline & & & Case 1 & Case 2 & Case 1 & Case 2 & Case 1 & Case 2 & Case 1 & Case 2 & Case 1 & Case 2 \\
\hline \multirow[t]{30}{*}{$C-F$} & -0.6 & 1 & 5.24863 & 5.66395 & 4.71243 & 6.50997 & 4.37675 & 6.86693 & 4.22066 & 6.72950 & 4.42416 & 6.26775 \\
\hline & & 2 & 20.6782 & 21.0110 & 20.0040 & 21.9602 & 19.4586 & 22.7719 & 22.7719 & 23.4807 & 17.8584 & 23.5657 \\
\hline & & 3 & 50.3367 & 49.2191 & 49.8027 & 50.4430 & 48.8317 & 52.1948 & 47.1362 & 54.0286 & 44.5712 & 55.3852 \\
\hline & & 4 & 94.8355 & 90.4947 & 94.4329 & 92.4857 & 92.6090 & 95.7134 & 89.4361 & 98.9904 & 85.1080 & 101.883 \\
\hline & & 5 & 156.055 & 145.153 & 154.034 & 148.298 & 150.930 & 153.537 & 145.763 & 158.611 & 140.205 & 163.268 \\
\hline & -0.4 & 1 & 4.47769 & 4.99796 & 3.98421 & 5.73764 & 3.69879 & 6.02308 & 3.57797 & 5.87318 & 3.86578 & 5.4441 \\
\hline & & 2 & 21.8076 & 22.0237 & 21.0035 & 23.1441 & 20.4178 & 23.9995 & 19.6468 & 24.6895 & 19.9372 & 24.7168 \\
\hline & & 3 & 56.5137 & 55.3678 & 55.9637 & 56.5601 & 54.8844 & 58.5515 & 52.9266 & 60.6072 & 57.3247 & 62.0384 \\
\hline & & 4 & 108.434 & 104.582 & 108.535 & 106.177 & 106.403 & 109.940 & 102.654 & 113.718 & 105.458 & 116.900 \\
\hline & & 5 & 180.932 & 168.481 & 178.831 & 172.181 & 175.119 & 178.346 & 168.971 & 184.244 & 166.759 & 189.462 \\
\hline & -0.2 & 1 & 3.96618 & 4.56252 & 3.49561 & 5.21665 & 3.24952 & 5.44536 & 3.15675 & 5.28434 & 3.51417 & 4.88086 \\
\hline & & 2 & 22.8699 & 23.3752 & 22.0423 & 24.3356 & 21.4026 & 25.2486 & 20.5870 & 25.9309 & 21.6115 & 25.9061 \\
\hline & & 3 & 62.8528 & 61.0208 & 61.8333 & 62.3096 & 60.6416 & 64.5452 & 58.4149 & 66.8288 & 62.9876 & 68.3468 \\
\hline & & 4 & 121.556 & 116.251 & 121.676 & 118.852 & 119.243 & 123.129 & 114.938 & 127.389 & 130.355 & 130.857 \\
\hline & & 5 & 202.072 & 189.284 & 201.711 & 194.147 & 197.421 & 201.178 & 190.349 & 207.850 & 225.875 & 213.596 \\
\hline & 0.0 & 1 & 3.63209 & 4.23374 & 3.14051 & 4.83168 & 2.92561 & 5.01564 & 2.85446 & 4.84663 & 3.10906 & 4.46439 \\
\hline & & 2 & 23.6312 & 24.7248 & 23.0523 & 25.4922 & 22.3538 & 26.4648 & 21.4949 & 27.1414 & 22.2552 & 27.0680 \\
\hline & & 3 & 68.2915 & 66.1484 & 67.4751 & 67.7866 & 66.1692 & 70.2669 & 63.6716 & 72.7786 & 66.7292 & 74.387 \\
\hline & & 4 & 135.212 & 128.603 & 134.154 & 130.834 & 131.425 & 135.611 & 126.577 & 140.338 & 137.669 & 144.080 \\
\hline & & 5 & 225.468 & 208.583 & 223.312 & 214.840 & 218.462 & 222.698 & 210.506 & 230.107 & 238.091 & 236.355 \\
\hline & 0.2 & 1 & 3.36348 & 3.97340 & 2.86822 & 4.53043 & 2.67867 & 4.67861 & 2.62432 & 4.50426 & 2.79864 & 4.14012 \\
\hline & & 2 & 24.4510 & 25.6833 & 24.0188 & 26.6073 & 23.2602 & 27.6376 & 22.3619 & 28.3074 & 22.8564 & 28.1876 \\
\hline & & 3 & 75.2230 & 70.6983 & 72.9399 & 73.0562 & 71.5191 & 75.7805 & 68.7499 & 78.5189 & 70.6037 & 80.2199 \\
\hline & & 4 & 149.469 & 146.281 & 146.146 & 142.308 & 143.123 & 147.574 & 137.742 & 152.755 & 145.524 & 156.764 \\
\hline & & 5 & 239.044 & 302.382 & 243.985 & 234.611 & 238.588 & 243.269 & 229.776 & 251.387 & 249.979 & 258.115 \\
\hline & 0.4 & 1 & 3.11747 & 3.54799 & 2.65117 & 4.28528 & 2.48280 & 4.40441 & 2.44172 & 4.22666 & 2.55922 & 3.87825 \\
\hline & & 2 & 25.9216 & 27.5856 & 24.9404 & 27.6825 & 24.1220 & 28.7669 & 23.1893 & 29.4278 & 23.4731 & 29.2636 \\
\hline & & 3 & 80.4953 & 74.6903 & 78.2643 & 78.1623 & 76.7284 & 81.1298 & 73.6872 & 84.0933 & 74.5624 & 85.8872 \\
\hline & & 4 & 156.165 & 260.079 & 157.761 & 153.390 & 154.444 & 159.137 & 148.540 & 164.761 & 153.999 & 169.029 \\
\hline & & 5 & 263.324 & 370.126 & 263.946 & 253.675 & 258.011 & 263.111 & 248.365 & 271.917 & 263.455 & 279.107 \\
\hline
\end{tabular}


Table 12: Non-dimensional natural frequencies $\Omega_{n}$ of an axially functionally graded and nonuniform cross-section simply supported beam with $A(x)=(1+\beta x)^{2}$ and $I(x)=(1+\beta x)^{4}$, and $E(x)$ and $\rho(x)$ changing in accordance with 17 and 18

\begin{tabular}{|c|c|c|c|c|c|c|c|c|c|c|c|c|}
\hline & \multirow{3}{*}{$\beta$} & \multirow{3}{*}{ Mode } & \multicolumn{10}{|c|}{$\alpha$} \\
\hline & & & \multicolumn{2}{|c|}{-10} & \multicolumn{2}{|c|}{-3} & \multicolumn{2}{|c|}{0} & \multicolumn{2}{|c|}{3} & \multicolumn{2}{|c|}{10} \\
\hline & & & Case 1 & Case 2 & Case 1 & Case 2 & Case 1 & Case 2 & Case 1 & Case 2 & Case 1 & Case 2 \\
\hline \multirow[t]{30}{*}{$S-S$} & -0.6 & 1 & 7.25496 & 6.27605 & 7.28940 & 6.21801 & 7.19290 & 6.45971 & 6.89874 & 6.77262 & 6.41542 & 7.10246 \\
\hline & & 2 & 31.1844 & 27.5181 & 30.5010 & 28.6164 & 29.5438 & 29.8912 & 28.4334 & 30.5705 & 27.3279 & 30.9338 \\
\hline & & 3 & 69.5049 & 61.8984 & 68.0843 & 63.8432 & 66.1358 & 66.4256 & 63.7265 & 68.1800 & 60.9766 & 69.1615 \\
\hline & & 4 & 122.863 & 110.685 & 120.496 & 112.767 & 117.221 & 117.146 & 113.027 & 120.401 & 107.935 & 122.462 \\
\hline & & 5 & 191.313 & 177.037 & 187.806 & 175.496 & 182.845 & 182.186 & 176.367 & 187.340 & 161.089 & 190.740 \\
\hline & -0.4 & 1 & 8.89776 & 7.72741 & 8.85331 & 7.79734 & 8.65017 & 8.14671 & 8.26256 & 8.50296 & 7.78256 & 8.80140 \\
\hline & & 2 & 36.3025 & 32.0805 & 35.5434 & 33.3036 & 34.5075 & 34.7140 & 33.2038 & 35.5687 & 31.7405 & 36.1189 \\
\hline & & 3 & 81.3263 & 72.5706 & 79.7036 & 74.7443 & 77.4950 & 77.6798 & 74.6752 & 79.7541 & 71.5480 & 81.0578 \\
\hline & & 4 & 144.172 & 129.806 & 141.463 & 132.538 & 137.649 & 137.598 & 132.713 & 141.392 & 125.297 & 143.879 \\
\hline & & 5 & 224.884 & 209.699 & 220.842 & 206.745 & 214.979 & 214.543 & 207.324 & 220.528 & 194.146 & 224.549 \\
\hline & -0.2 & 1 & 10.2669 & 8.95252 & 10.1383 & 9.15491 & 9.84220 & 9.58773 & 9.38966 & 9.96201 & 8.93653 & 10.2188 \\
\hline & & 2 & 41.1055 & 36.2726 & 40.2920 & 37.7238 & 39.1778 & 39.2763 & 37.6780 & 40.3008 & 35.9936 & 41.0045 \\
\hline & & 3 & 92.3162 & 82.2952 & 90.5167 & 84.8736 & 88.0613 & 88.1522 & 84.8407 & 90.5396 & 81.2987 & 92.1251 \\
\hline & & 4 & 163.910 & 145.777 & 160.808 & 150.766 & 156.490 & 156.465 & 150.854 & 160.771 & 143.342 & 163.661 \\
\hline & & 5 & 255.791 & 229.961 & 251.166 & 235.431 & 244.466 & 244.249 & 235.720 & 251.009 & 223.400 & 255.592 \\
\hline & 0.0 & 1 & 11.4625 & 10.0301 & 11.2443 & 10.3669 & 10.8663 & 10.8663 & 10.3669 & 11.2443 & 10.0322 & 11.4623 \\
\hline & & 2 & 45.7174 & 40.2024 & 44.8537 & 41.9681 & 43.6638 & 43.6638 & 41.9681 & 44.8537 & 40.2049 & 45.7191 \\
\hline & & 3 & 102.701 & 91.1871 & 100.811 & 94.5037 & 98.1177 & 98.1177 & 94.5037 & 100.812 & 91.1415 & 102.704 \\
\hline & & 4 & 182.505 & 158.809 & 179.109 & 167.999 & 174.309 & 174.309 & 167.999 & 179.109 & 158.752 & 182.481 \\
\hline & & 5 & 284.975 & 240.013 & 279.745 & 262.468 & 272.250 & 272.250 & 262.468 & 279.745 & 240.272 & 284.985 \\
\hline & 0.2 & 1 & 12.5243 & 11.0063 & 12.2236 & 11.4737 & 11.7727 & 12.0274 & 11.2388 & 12.3999 & 10.8700 & 12.5686 \\
\hline & & 2 & 50.1747 & 43.9139 & 49.2845 & 46.0847 & 48.0217 & 47.9236 & 46.1301 & 49.2760 & 44.0152 & 50.2408 \\
\hline & & 3 & 112.850 & 99.5920 & 110.739 & 103.777 & 107.812 & 107.721 & 103.810 & 110.716 & 99.4961 & 112.8049 \\
\hline & & 4 & 200.535 & 170.229 & 196.668 & 184.525 & 191.399 & 191.425 & 184.437 & 196.705 & 176.742 & 200.338 \\
\hline & & 5 & 312.533 & 252.314 & 307.082 & 288.332 & 298.821 & 299.038 & 288.043 & 307.240 & 276.450 & 312.856 \\
\hline & 0.4 & 1 & 13.4962 & 11.9115 & 13.1073 & 12.5001 & 12.5911 & 13.0984 & 12.0319 & 13.4592 & 11.7095 & 13.5845 \\
\hline & & 2 & 54.5467 & 47.4764 & 53.6179 & 50.1028 & 52.2846 & 52.0848 & 50.1968 & 53.5980 & 47.8578 & 54.6819 \\
\hline & & 3 & 122.512 & 107.629 & 120.389 & 112.780 & 117.233 & 117.050 & 112.847 & 120.341 & 108.144 & 122.664 \\
\hline & & 4 & 217.773 & 181.455 & 213.664 & 200.514 & 207.939 & 207.990 & 200.338 & 213.738 & 192.316 & 217.721 \\
\hline & & 5 & 339.974 & 268.653 & 333.480 & 313.308 & 324.473 & 324.908 & 312.729 & 333.794 & 300.969 & 339.895 \\
\hline
\end{tabular}


Table 13: Non-dimensional natural frequencies $\Omega_{n}$ of an axially functionally graded and nonuniform cross-section clamped-pinned beam with $A(x)=(1+\beta x)^{2}$ and $I(x)=(1+\beta x)^{4}$, and $E(x)$ and $\rho(x)$ changing in accordance with 17 and 18)

\begin{tabular}{|c|c|c|c|c|c|c|c|c|c|c|c|c|}
\hline & \multirow{3}{*}{$\beta$} & \multirow{3}{*}{ Mode } & \multicolumn{10}{|c|}{$\alpha$} \\
\hline & & & \multicolumn{2}{|c|}{-10} & \multicolumn{2}{|c|}{-3} & \multicolumn{2}{|c|}{0} & \multicolumn{2}{|c|}{3} & \multicolumn{2}{|c|}{10} \\
\hline & & & Case 1 & Case 2 & Case 1 & Case 2 & Case 1 & Case 2 & Case 1 & Case 2 & Case 1 & Case 2 \\
\hline \multirow[t]{30}{*}{$C-P$} & -0.6 & 1 & 12.9193 & 13.7797 & 12.4695 & 14.4721 & 12.4513 & 14.3477 & 12.5458 & 14.1075 & 12.4197 & 14.1012 \\
\hline & & 2 & 38.9033 & 38.6426 & 38.3811 & 39.5267 & 37.9677 & 40.2060 & 37.3309 & 40.6393 & 36.0462 & 40.9910 \\
\hline & & 3 & 80.0231 & 76.5424 & 79.2618 & 78.1420 & 78.0815 & 80.1744 & 76.1066 & 81.8057 & 72.3270 & 82.9396 \\
\hline & & 4 & 136.370 & 127.832 & 135.110 & 130.501 & 132.743 & 134.420 & 128.905 & 137.656 & 120.597 & 139.957 \\
\hline & & 5 & 207.966 & 192.950 & 205.936 & 196.686 & 201.968 & 203.020 & 195.752 & 208.260 & 177.064 & 212.038 \\
\hline & -0.4 & 1 & 14.2144 & 15.1079 & 13.8179 & 15.7182 & 13.7694 & 15.6379 & 13.7661 & 15.4944 & 13.5657 & 15.5339 \\
\hline & & 2 & 44.6125 & 44.1992 & 44.1116 & 45.0560 & 43.6753 & 45.8430 & 42.879 & 46.4377 & 41.2507 & 47.0013 \\
\hline & & 3 & 93.0592 & 88.8644 & 92.2442 & 90.6095 & 90.8761 & 92.9839 & 88.5003 & 94.9406 & 84.1295 & 96.4028 \\
\hline & & 4 & 159.556 & 149.551 & 158.106 & 152.519 & 155.278 & 157.126 & 150.687 & 160.925 & 140.008 & 163.682 \\
\hline & & 5 & 243.998 & 227.280 & 241.665 & 230.845 & 236.872 & 238.315 & 229.445 & 244.426 & 204.932 & 248.841 \\
\hline & -0.2 & 1 & 15.3578 & 16.2641 & 14.9846 & 16.8374 & 14.8876 & 16.8181 & 14.8008 & 16.7429 & 14.6032 & 16.7884 \\
\hline & & 2 & 49.9003 & 49.2813 & 49.4237 & 50.1377 & 48.9538 & 51.0479 & 47.9790 & 51.8091 & 46.2008 & 52.5517 \\
\hline & & 3 & 105.089 & 100.233 & 104.210 & 102.038 & 102.653 & 104.750 & 99.8803 & 107.029 & 95.5192 & 108.789 \\
\hline & & 4 & 180.910 & 169.133 & 179.197 & 172.657 & 175.927 & 177.912 & 170.621 & 182.247 & 160.805 & 185.432 \\
\hline & & 5 & 276.925 & 259.249 & 274.333 & 262.039 & 268.763 & 270.557 & 260.210 & 277.480 & 237.082 & 282.484 \\
\hline & 0.0 & 1 & 16.4002 & 17.3141 & 16.0255 & 17.8670 & 15.8734 & 17.9148 & 15.7169 & 17.8873 & 15.5435 & 17.9201 \\
\hline & & 2 & 54.9317 & 54.0109 & 54.4677 & 54.9298 & 53.9586 & 55.9710 & 52.8020 & 56.8997 & 50.8730 & 57.8023 \\
\hline & & 3 & 116.374 & 111.047 & 115.517 & 112.794 & 113.770 & 115.840 & 110.605 & 118.435 & 106.244 & 120.473 \\
\hline & & 4 & 200.920 & 186.874 & 199.056 & 191.578 & 195.356 & 197.456 & 189.361 & 202.307 & 180.689 & 205.897 \\
\hline & & 5 & 308.074 & 290.924 & 305.024 & 291.318 & 298.708 & 300.829 & 289.084 & 308.522 & 270.827 & 314.084 \\
\hline & 0.2 & 1 & 17.3490 & 18.3014 & 16.9720 & 18.8380 & 16.7634 & 18.9450 & 16.5486 & 18.9503 & 16.4064 & 18.9608 \\
\hline & & 2 & 59.7676 & 58.4471 & 59.3224 & 59.5157 & 58.7708 & 60.6922 & 57.4282 & 61.7884 & 55.3210 & 62.8390 \\
\hline & & 3 & 127.349 & 121.578 & 126.356 & 123.068 & 124.417 & 126.444 & 120.863 & 129.352 & 116.383 & 131.654 \\
\hline & & 4 & 220.271 & 202.588 & 218.036 & 209.629 & 213.912 & 216.112 & 207.247 & 221.464 & 199.242 & 225.441 \\
\hline & & 5 & 337.244 & 323.997 & 334.304 & 319.232 & 327.262 & 329.695 & 316.608 & 338.128 & 302.651 & 344.223 \\
\hline & 0.4 & 1 & 18.2423 & 19.2524 & 17.8444 & 19.7553 & 17.5798 & 19.9208 & 17.3166 & 19.9475 & 17.2087 & 19.9302 \\
\hline & & 2 & 64.4810 & 62.6023 & 64.0353 & 63.9453 & 63.4388 & 65.2602 & 61.9070 & 66.5238 & 59.5994 & 67.7141 \\
\hline & & 3 & 137.723 & 131.998 & 136.839 & 132.976 & 134.707 & 136.681 & 130.766 & 139.897 & 126.073 & 142.451 \\
\hline & & 4 & 238.763 & 216.519 & 236.348 & 227.021 & 231.805 & 234.095 & 224.484 & 239.935 & 216.717 & 244.284 \\
\hline & & 5 & 366.410 & 362.219 & 362.513 & 346.108 & 354.760 & 357.494 & 343.107 & 366.643 & 331.697 & 373.251 \\
\hline
\end{tabular}


Table 14: Non-dimensional natural frequencies $\Omega_{n}$ of an axially functionally graded and nonuniform cross-section fully clamped beam with $A(x)=(1+\beta x)^{2}$ and $I(x)=(1+\beta x)^{4}$, and $E(x)$ and $\rho(x)$ changing in accordance with 17 and 18

\begin{tabular}{|c|c|c|c|c|c|c|c|c|c|c|c|c|}
\hline & \multirow{3}{*}{$\beta$} & \multicolumn{11}{|c|}{$\alpha$} \\
\hline & & \multirow[t]{2}{*}{ Mode } & \multicolumn{2}{|c|}{-10} & \multicolumn{2}{|c|}{-3} & \multicolumn{2}{|c|}{0} & \multicolumn{2}{|c|}{3} & \multicolumn{2}{|c|}{10} \\
\hline & & & Case 1 & Case 2 & Case 1 & Case 2 & Case 1 & Case 2 & Case 1 & Case 2 & Case 1 & Case 2 \\
\hline \multirow[t]{30}{*}{$C-C$} & -0.6 & 1 & 16.0941 & 17.0724 & 15.7308 & 17.6020 & 15.9232 & 17.2096 & 16.3018 & 16.7718 & 16.4644 & 16.6712 \\
\hline & & 2 & 45.5124 & 44.9199 & 45.0176 & 45.7723 & 44.7470 & 46.2067 & 44.3980 & 46.2577 & 43.8359 & 46.2752 \\
\hline & & 3 & 90.3178 & 86.0362 & 89.5264 & 87.7129 & 88.3928 & 89.5854 & 86.6294 & 90.7248 & 84.8628 & 91.1810 \\
\hline & & 4 & 150.404 & 140.630 & 149.050 & 143.462 & 146.630 & 147.324 & 142.906 & 150.019 & 135.297 & 151.435 \\
\hline & & 5 & 225.756 & 209.349 & 223.574 & 213.069 & 219.455 & 219.458 & 213.244 & 224.146 & 195.689 & 226.694 \\
\hline & -0.4 & 1 & 18.8783 & 19.8346 & 18.6189 & 20.1879 & 18.8965 & 19.7273 & 19.3310 & 19.3019 & 19.4258 & 19.2732 \\
\hline & & 2 & 53.5683 & 52.6230 & 53.1176 & 53.4274 & 52.8930 & 53.8611 & 52.5145 & 53.9365 & 51.9323 & 54.0689 \\
\hline & & 3 & 106.495 & 101.225 & 105.653 & 103.040 & 104.366 & 105.161 & 102.318 & 106.445 & 100.635 & 107.060 \\
\hline & & 4 & 177.470 & 165.970 & 175.916 & 169.034 & 173.044 & 173.508 & 168.662 & 176.557 & 162.530 & 178.130 \\
\hline & & 5 & 266.478 & 248.095 & 263.881 & 251.458 & 258.922 & 258.927 & 251.574 & 264.259 & 244.036 & 267.131 \\
\hline & -0.2 & 1 & 21.5180 & 22.3963 & 21.3364 & 22.6145 & 21.6912 & 22.1015 & 22.1877 & 21.6759 & 22.1918 & 21.7179 \\
\hline & & 2 & 61.0333 & 59.6901 & 60.6269 & 60.4651 & 60.4349 & 60.9180 & 60.0093 & 61.0350 & 59.3664 & 61.2832 \\
\hline & & 3 & 121.353 & 115.127 & 120.468 & 117.056 & 119.025 & 119.423 & 116.695 & 120.863 & 114.858 & 121.628 \\
\hline & & 4 & 202.253 & 189.014 & 200.485 & 192.367 & 197.180 & 197.413 & 192.181 & 200.804 & 187.796 & 202.566 \\
\hline & & 5 & 303.693 & 283.659 & 300.647 & 286.446 & 294.900 & 294.903 & 286.504 & 300.836 & 284.301 & 304.007 \\
\hline & 0.0 & 1 & 24.0629 & 24.8279 & 23.9433 & 24.9364 & 24.3754 & 24.3754 & 24.9364 & 23.9433 & 24.8283 & 24.0629 \\
\hline & & 2 & 68.1190 & 66.3359 & 67.7553 & 67.1041 & 67.5875 & 67.5875 & 67.1041 & 67.7553 & 66.3363 & 68.1192 \\
\hline & & 3 & 135.351 & 128.158 & 134.439 & 130.228 & 132.839 & 132.839 & 130.228 & 134.439 & 128.152 & 135.352 \\
\hline & & 4 & 225.540 & 210.309 & 223.579 & 214.262 & 219.855 & 219.855 & 214.262 & 223.579 & 210.300 & 225.536 \\
\hline & & 5 & 338.605 & 317.091 & 335.144 & 319.253 & 328.641 & 328.641 & 319.253 & 335.144 & 317.100 & 338.604 \\
\hline & 0.2 & 1 & 26.5366 & 27.1668 & 26.4723 & 27.1827 & 26.9839 & 26.5740 & 27.6093 & 26.1329 & 27.3717 & 26.3381 \\
\hline & & 2 & 74.9315 & 72.6668 & 74.6131 & 73.4587 & 74.4633 & 73.9802 & 73.9145 & 74.2051 & 72.9893 & 74.6847 \\
\hline & & 3 & 148.777 & 140.559 & 147.811 & 142.801 & 146.051 & 145.654 & 143.162 & 147.416 & 140.813 & 148.475 \\
\hline & & 4 & 247.821 & 230.039 & 245.625 & 235.136 & 241.489 & 241.256 & 235.322 & 245.306 & 231.192 & 247.457 \\
\hline & & 5 & 371.844 & 347.994 & 368.027 & 350.510 & 360.791 & 360.788 & 350.453 & 367.838 & 347.282 & 371.580 \\
\hline & 0.4 & 1 & 28.9584 & 29.4358 & 28.9432 & 29.3711 & 29.5375 & 28.7138 & 30.2256 & 28.2631 & 29.8445 & 28.5613 \\
\hline & & 2 & 81.5429 & 78.7483 & 81.2668 & 79.5978 & 81.1300 & 80.1633 & 80.5099 & 80.4498 & 79.4116 & 81.0453 \\
\hline & & 3 & 161.708 & 152.446 & 160.729 & 154.919 & 158.807 & 158.013 & 155.641 & 159.938 & 153.010 & 161.143 \\
\hline & & 4 & 269.260 & 248.332 & 266.878 & 255.235 & 262.334 & 261.869 & 255.607 & 266.239 & 251.098 & 268.583 \\
\hline & & 5 & 404.054 & 375.904 & 399.688 & 380.593 & 391.736 & 391.730 & 380.477 & 399.310 & 376.170 & 403.327 \\
\hline
\end{tabular}




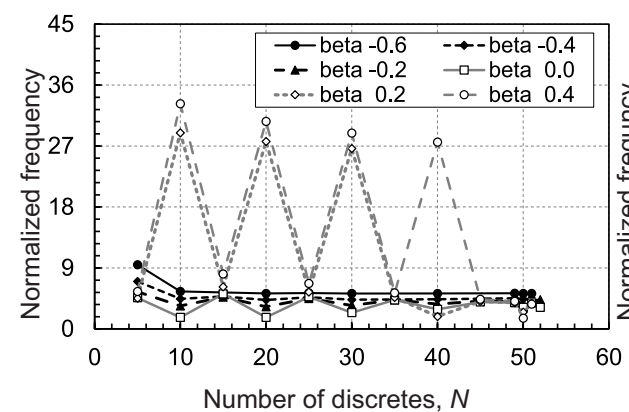

(a)

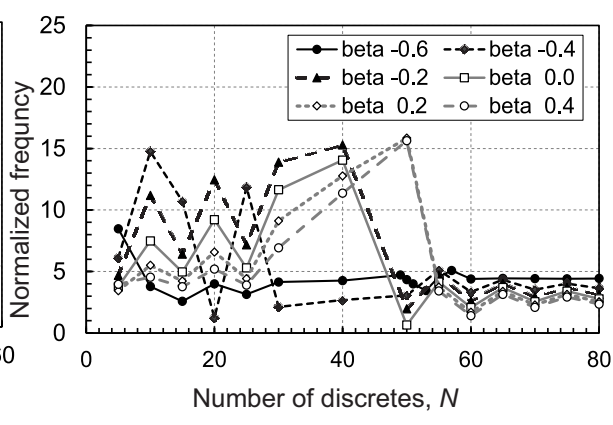

(b)

Figure 7: Convergence of the fundamental normalized frequencies for the C-F beam in the case 1: (a) $\alpha=-10$; and (b) $\alpha=10$.

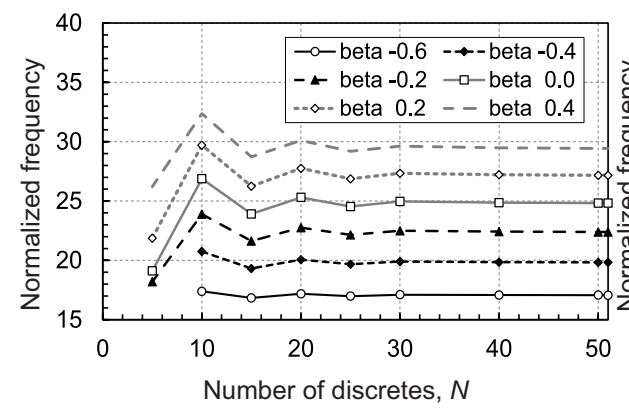

(a)

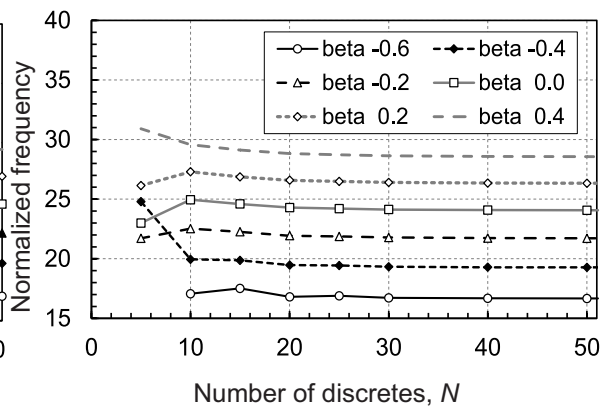

(b)

Figure 8: Convergence of the fundamental normalized frequencies for the $\mathrm{C}-\mathrm{C}$ beam in the case 2: (a) $\alpha=-10$; and (b) $\alpha=10$.

mode frequencies of such AFGM non-uniform beams often do not follow the variation tendency which is established for their fundamental frequencies and each mode may have its own variation law. Moreover, the variations of frequencies with changing $\alpha$ and $\beta$ are different for the material gradation patterns called as case 1 and case 2 , and for symmetric boundary conditions the natural frequencies, in general, show opposite trends in these two cases.

The results obtained by the proposed approach implementing the DTM demonstrate its good versatility and efficiency for solving the free vibration problem of beams for a variety of cross-sectional non-uniformity, axial mate- 
rial inhomogeneity and boundary conditions. Moreover, using the results of the convergence analyses of the obtained DTM solutions, we have proven a high enough accuracy of the developed algorithm as well as have established some issues that may happen in the computations of the natural frequencies of axially functionally graded beams with non-uniform cross-section subjected to different end supports. Therefore, these results can be considered as benchmarks for other researchers.

\section{Conclusions}

The differential transform method is effectively applied to the free vibration problem of axially functionally graded non-uniform cross-section Euler-Bernoulli beams with arbitrary gradation profiles and non-uniform cross-sectional parameters. The method provides an effective way for solving the governing differential beam equation with arbitrarily varying coefficients. For various beam configurations and different boundary conditions, we have used the differential transformations to reduce the differential beam equation to a set of recurrent algebraic equations. This set of equations together with appropriately transformed boundary conditions results in to a polynomial eigenvalue problem that allows us to find required natural frequencies. By comparing the results of calculations carried out with the DTM and those available in the literature for various axially graded and non-uniform cross-section beams subjected to different end supports, effectiveness and versatility of the DTM has been demonstrated. The superiority of the DTM to other numerical methods has been also shown by its capability to treat any arbitrary gradation profiles and cross-sectional non-uniformities. This has allowed us to demonstrate the influence of the in-

homogeneity and non-uniformity parameters on the natural frequencies of the metal/ceramic $\mathrm{AlZrO}_{2}$ beams.

The accuracy of the method is proven by supplement studies concerning the convergence of solutions. We have concluded that the fastest convergence of the DTM is at the centre of approximation interval and as one moves away from the 
centre, the rate of convergence slows down dramatically. Furthermore, we have observed that though the DTM provides very accurate results for most of cases studied in the paper, some computational issues arise when the DTM is applied to the problems which feature high material gradients. Hence, a convergence analysis is always needed to ensure the reliability of the solution and to avoid inaccuracy of results. Based on the revealed advantages and drawbacks of the DTM solutions we would like to believe that the results presented in this paper can be useful for other researchers and can be used by them as references to validate their results.

\section{Acknowledgements}

This research has been done under financial support of the Erasmus Mundus post-doc exchange program ACTIVE, Grant Agreement No. 2013-2523/001-001 at the University of Southampton.

\section{Appendix A}

The coefficients $B_{K}(\omega), C_{K}(\omega), G_{K}(\omega)$ and $H_{K}(\omega)$ denoted in (8) are presented by the following recurrent expressions:

$$
\begin{aligned}
B_{K}(\omega) & =\frac{1}{(K+1)(K+2)(K+3)(K+4)} . \\
& {\left[\omega^{2}\left(\sum_{p=0}^{K-4} B_{p} M(K-4-p)+M(K)\right)-\right.} \\
& \sum_{p=0}^{K-1}(p+2)(p+3)(p+4) B_{p} D_{1}(K-1-p)- \\
& \left.\sum_{p=0}^{K-2}(p+3)(p+4) B_{p} D_{2}(K-2-p)\right],
\end{aligned}
$$




$$
\begin{aligned}
& C_{K}(\omega)=\frac{1}{(K+1)(K+2)(K+3)(K+4)} . \\
& {\left[\omega^{2}\left(\sum_{p=0}^{K-4} C_{p} M(K-4-p)+M(K-1)\right)-\right.} \\
& \sum_{p=0}^{K-1}(p+2)(p+3)(p+4) C_{p} D_{1}(K-1-p)- \\
& \left.\sum_{p=0}^{K-2}(p+3)(p+4) C_{p} D_{2}(K-2-p)\right] \text {, } \\
& G_{K}(\omega)=\frac{1}{(K+1)(K+2)(K+3)(K+4)} . \\
& {\left[\omega^{2}\left(\sum_{p=0}^{K-4} G_{p} M(K-4-p)+M(K-2)\right)-\right.} \\
& \sum_{p=0}^{K-1}(p+2)(p+3)(p+4) G_{p} D_{1}(K-1-p)- \\
& \left.\left(\sum_{p=0}^{K-2}(p+3)(p+4) G_{p} D_{2}(K-2-p)+2 D_{2}(K)\right)\right], \\
& H_{K}(\omega)=\frac{1}{(K+1)(K+2)(K+3)(K+4)} . \\
& {\left[\omega^{2}\left(\sum_{p=0}^{K-4} H_{p} M(K-4-p)+M(K-3)\right)-\right.} \\
& \left(\sum_{p=0}^{K-1}(p+2)(p+3)(p+4) H_{p} D_{1}(K-1-p)+6 D_{1}(K)\right)- \\
& \left.\left(\sum_{p=0}^{K-2}(p+3)(p+4) H_{p} D_{2}(K-2-p)+6 D_{2}(K-1)\right)\right]
\end{aligned}
$$

\section{Appendix B}

The constants taken from [19] for calculations of the natural frequencies presented in Tables 46 have been used in the following forms: 


$$
\begin{gathered}
b_{00}=26 a_{0}, b_{01}=16 a_{0}, b_{02}=6 a_{0}, b_{03}=-4 a_{0}, b_{04}=a_{0}, \text { where } a_{0}=1 \\
b_{10}=\frac{2\left(71 a_{1}+91 a_{0}\right)}{5}, b_{11}=\frac{2\left(51 a_{1}+56 a_{0}\right)}{5}, b_{12}=\frac{2\left(31 a_{1}+21 a_{0}\right)}{5}, \\
\quad b_{13}=\frac{2\left(11 a_{1}-14 a_{0}\right)}{5}, b_{14}=\frac{-18 a_{1}+7 a_{0}}{5}, b_{15}=a_{1} \\
\text { where } a_{0}=1, a_{1}=1 \\
b_{20}=\frac{465 a_{2}+568 a_{1}+728 a_{0}}{15}, b_{21}=\frac{2\left(181 a_{2}+204 a_{1}+224 a_{0}\right)}{15} \\
b_{22}=\frac{259 a_{2}+248 a_{1}+168 a_{0}}{15}, b_{23}=\frac{4\left(39 a_{2}+22 a_{1}-28 a_{0}\right)}{15} \\
b_{24}=\frac{53 a_{2}-72 a_{1}+28 a_{0}}{5}, b_{25}=\frac{2\left(-5 a_{2}+2 a_{1}\right)}{3}, b_{26}=a_{2} \\
\text { where } a_{0}=1.5954, a_{1}=0.04, a_{2}=1
\end{gathered}
$$

\section{References}

[1] Y. Miyamoto, W. Kaysser, B. Rabin, A. Kawasaki, R. Ford, Functionally Graded Materials: Design, Processing and Applications, Springer US, New York, 1999. doi:10.1007/978-1-4615-5301-4.

URL http://www . springer . com/gb/book/9780412607608

[2] T. Sadowski, Non-symmetric thermal shock in ceramic matrix composite (CMC) materials, in: R. de Borst, T. Sadowski (Eds.), Lecture Notes on Composite Materials - Current Topics and Achievements, Vol. 154 of Solid Mechanics and Its Applications, Springer Netherlands, 2009, pp. 99-148. doi:10.1007/978-1-4020-8772-1.

[3] V. N. Burlayenko, H. Altenbach, T. Sadowski, S. D. Dimitrova, Computational simulations of thermal shock cracking by the virtual crack closure technique in a functionally graded plate, Computational Materials Science 116 (15) (2016) 11-21. doi:http://dx.doi.org/10.1016/j.commatsci. 2015.08 .038

[4] V. N. Burlayenko, Modelling thermal shock in functionally graded plates with finite element method, Advances in Materials Science and Engineering 
2016 (Article ID 7514638) (2016) 12 pages. doi:http://dx.doi.org/10. $1155 / 2016 / 7514638$

[5] M. Şimşek, Fundamental frequency analysis of functionally graded beams by using different higher-order beam theories, Nuclear Engineering and Design 240 (4) (2010) 697-705. doi:10.1016/j.nucengdes.2009.12.013

[6] S. Abrate, Vibration of non-uniform rods and beams, Journal of Sound and Vibration 185 (4) (1995) 703-716. doi:10.1006/jsvi.1995.0410.

[7] R. Attarnejad, S. J. Semnani, A. Shahba, Basic displacement functions for free vibration analysis of non-prismatic timoshenko beams, Finite Elements in Analysis and Design 46 (10) (2010) 916-929. doi:10.1016/j.finel. 2010.06 .005

[8] S. Q. Guo, S. P. Yang, Transverse vibrations of arbitrary non-uniform beams, Applied Mathematics and Mechanics (English Edition) 35 (5) (2014) 607-620.

[9] D. Garijo, Free vibration analysis of nonuniform eulerbernoulli beams by means of bernstein pseudospectral collocation, Engineering with Computers 31 (2015) 813-823. doi:110.1007/s00366-015-0401-6.

[10] I. Elishakoff, S. Candan, Apparently first closed-form solution for vibrating: Inhomogeneous beams, International Journal of Solids and Structures 38 (19) (2001) 3411-3441. doi:10.1016/S0020-7683(00)00266-3.

[11] S. Candan, I. Elishakoff, Apparently first closed-form solution for frequencies of deterministically and/or stochastically inhomogeneous simply supported beams, Journal of Applied Mechanics 68 (2001) 176-185. doi:10.1115/1.1355034.

[12] Q. S. Li, A new exact approach for determining natural frequencies and mode shapes of non-uniform shear beams with arbitrary distribution of mass or stiffness, International Journal of Solids and Structures 37 (37) (2000) 5123-5141. 
[13] H. Ait Atmane, A. Tounsi, S. A. Meftah, H. A. Belhadj, Free vibration behavior of exponential functionally graded beams with varying crosssection, Journal of Vibration and Control 17 (2) (2010) 311-318. doi: $10.1177 / 1077546310370691$

[14] X. F. Li, Y. A. Kang, J. X. Wu, Exact frequency equations of free vibration of exponentially functionally graded beams, Applied Acoustics 74 (3) (2013) 413-420.

[15] A. Y. Tang, J. X. Wu, X. F. Li, K. Y. Lee, Exact frequency equations of free vibration of exponentially non-uniform functionally graded timoshenko beams, International Journal of Mechanical Sciences 89 (2014) 1-11. doi: 10.1016/j.ijmecsci.2014.08.017.

[16] A. E. Alshorbagy, M. A. Eltaher, F. F. Mahmoud, Free vibration characteristics of a functionally graded beam by finite element method, Applied Mathematical Modelling 35 (1) (2011) 412-425. doi:10.1016/j. apm.2010.07.006.

[17] A. Shahba, R. Attarnejad, M. T. Marvi, S. Hajilar, Free vibration and stability analysis of axially functionally graded tapered timoshenko beams with classical and non-classical boundary conditions, Composites Part B: Engineering 42 (4) (2011) 801-808. doi:10.1016/j.compositesb.2011. 01.017 .

[18] V. N. Burlayenko, H. Altenbach, T. Sadowski, S. D. Dimitrova, A. Bhaskar, Modelling functionally graded materials in heat transfer and thermal stress analysis by means of graded finite elements, Applied Mathematical Modelling 45 (2017) 422-438. doi:10.1016/j.apm.2017.01.005.

[19] Y. Huang, X.-F. Li, A new approach for free vibration of axially functionally graded beams with non-uniform cross-section, Journal of Sound and Vibration 329 (11) (2010) 2291-2303. doi:10.1016/j.jsv.2009.12.029. 
[20] H. Hein, L. Feklistova, Free vibrations of non-uniform and axially functionally graded beams using haar wavelets, Engineering Structures 33 (12) (2011) 3696-3701. doi:10.1016/j.engstruct.2011.08.006

[21] D. V. Bambill, D. H. Felix, R. E. Rossi, Vibration analysis of rotating timoshenko beams by means of the differential quadrature method, Structural Engineering and Mechanics 34 (2) (2010) 231-245.

[22] S. Rajasekaran, Differential transformation and differential quadrature methods for centrifugally stiffened axially functionally graded tapered beams, International Journal of Mechanical Sciences 74 (2013) 15-31. doi:10.1016/j.ijmecsci.2013.04.004.

[23] G. E. Pukhov, Computational structure for solving differential equations by taylor transformations, Cybernetics and Systems Analysis 14 (3) (1978) 383-390. doi:10.1007/BF01074670.

URL http://link.springer.com/article/10.1007 2FBF01074670?LI= true

[24] G. E. Pukhov, Taylor Transformations and Their Applications in Electrical and Electronics, Naukova Dumka, Kiev, 1978, (in Russian).

[25] G. E. Pukhov, Differential transforms and circuit-theory, International Journal of Circuit Theory and Applications 10 (3) (1982) 265-276. doi: $10.1002 /$ cta. 4490100307 .

[26] G. E. Pukhov, Differential Analysis of Circuits, Naukova Dumka, Kiev, 1982, (in Russian).

[27] G. E. Pukhov, Differential Transformations of Functions and Equations, Naukova Dumka, Kiev, 1980, (in Russian).

[28] G. E. Pukhov, Differential Transformations and Mathematical Modeling of Physical Processes, Naukova Dumka, Kiev, 1986, (in Russian). 
[29] G. E. Pukhov, Approximate Methods of Mathematical Modelling Based on the Use of Differential T-transformations, Naukova Dumka, Kiev, 1988, (in Russian).

[30] G. E. Pukhov, Differential spectrums and models, Naukova Dumka, Kiev, 1990, (in Russian).

[31] C. Bervillier, Status of the differential transformation method, Applied Mathematics and Computation 218 (20) (2012) 10158-10170. doi:10. $1016 / \mathrm{j}$. amc.2012.03.094.

[32] S. H. Simonyan, A. G. Avetisyan, Applied Theory of Differential Transforms, Chartaraget, Yerevan, 2010.

[33] A. G. Avetisyan, S. H. Simonyan, D. A. Ghazaryan, Solution of linear time optimal control problems in domain of differential transformations, Bulletin of Tomsk Politechnical University 315 (5) (2009) 5-13.

URL http://www.lib.tpu.ru/fulltext/v/Bulletin\{_\}TPU/2009/ v315/i5/01.pdf

[34] A. G. Avetisyan, V. R. Avinyan, D. A. Ghazaryan, A method for solving silvester type parametric matrix equation, Proceedings of the NAS RA and SEUA: Technical Sciences 66 (4) (2013) 376-383.

URL http://tech.asj-oa.am/3987/1/376.pdf

[35] O. O. Ozgumus, M. O. Kaya, Flapwise bending vibration analysis of double tapered rotating eulerbernoulli beam by using the differential transform method, Meccanica 41 (6) (2006) 661-670. doi:10.1007/ s11012-006-9012-z.

[36] C. Mei, Application of differential transformation technique to free vibration analysis of a centrifugally stiffened beam, Computers and Structures 86 (11-12) (2008) 1280-1284. doi:10.1016/j.compstruc.2007.10.003

[37] S. M. Abdelghany, K. M. Ewis, A. A. Mahmoud, M. M. Nassar, Vibration of a circular beam with variable cross sections using differential transforma- 
tion method, Beni-Suef University Journal of Basic and Applied Sciences 4 (2015) 185-191. doi:http://dx.doi.org/10.1016/j.bjbas.2015.05. 006.

[38] N. Wattanasakulpong, J. Charoensuk, Vibration characteristics of stepped beams made of fgm using differential transformation method, Meccanica 50 (2015) 1089-1101. doi:10.1007/s11012-014-0054-3.

[39] K. Suddounga, J. Charoensuka, N. Wattanasakulpong, Vibration response of stepped fgm beams with elastically end constraints using differential transformation method, Applied Acoustics 77 (2014) 20-28. doi:http: //dx.doi.org/10.1016/j.apacoust.2013.09.018

[40] A. Shahba, S. Rajasekaran, Free vibration and stability of tapered EulerBernoulli beams made of axially functionally graded materials, Applied Mathematical Modelling 36 (7) (2012) 3094-3111. doi:10.1016/j.apm. 2011.09 .073

[41] S. Rajasekaran, E. N. Tochaei, Free vibration analysis of axially functionally graded tapered Timoshenko beams using differential transformation element method and differential quadrature element method of lowest-order, Meccanica (49) (2014) 995-1009. doi:10.1007/s11012-013-9847-z.

[42] F. Ebrahimi, M. Mokhtari, Vibration analysis of spinning exponentially functionally graded timoshenko beams based on differential transform method, Proceedings of the Institution of Mechanical Engineers, Part G: Journal of Aerospace Engineering 229 (14) (2015) 2559-2571. doi: 10.1177/0954410015580801

URL http://pig.sagepub.com.proxy.bib.uottawa.ca/content/229/ 14/2559.abstract

[43] W. J. W., S. P. Timoshenko, D. H. Young, Vibration Problems in Engineering, 5th Edition, John Wiley \& Sons, Inc., New York, 1990. 
[44] H. M. Antia, Numerical Methods for Scientists and Engineer, 2nd Edition, Birkhäuser Verlag, Basel, Switzerland, 2002. 